\title{
Natural dark matter and light bosons with an alternative left-right symmetry
}

\author{
Mariana Frank, ${ }^{a}$ Benjamin Fuks ${ }^{b, c}$ and Özer Özdal ${ }^{a}$ \\ ${ }^{a}$ Department of Physics, Concordia University, \\ 7141 Sherbrooke St. West, Montreal, Quebec H4B 1R6, Canada \\ ${ }^{b}$ Laboratoire de Physique Théorique et Hautes Energies (LPTHE), \\ UMR 7589, Sorbonne Université et CNRS, 4 place Jussieu, 75252 Paris Cedex 05, France \\ ${ }^{c}$ Institut Universitaire de France, 103 boulevard Saint-Michel, \\ 75005 Paris, France \\ E-mail: mariana.frank@concordia.ca, fuks@lpthe.jussieu.fr, \\ ozer.ozdal@concordia.ca
}

ABSTRACT: We perform a consistent analysis of the alternative left-right symmetric model emerging from $E_{6}$ grand unification. We include a large set of theoretical and experimental constraints, with a particular emphasis on dark matter observables and collider signals. We show that the exotic neutrino inherent to this class of models, the scotino, is a viable candidate for dark matter satisfying relic density and direct detection constraints. This has strong implications on the scotino mass restricting it to lie in a narrow window, as well as on the spectrum of Higgs bosons, rendering it predictable, with a few light scalar, pseudoscalar and charged states. Moreover, we also show that the extra charged $W^{\prime}$ gauge boson can be light, and investigate the most promising signals at the future high-luminosity upgrade of the LHC. Our findings show that the most optimistic cosmologically-favoured scenarios should be observable at $5 \sigma$, whilst others could leave visible hints provided the background is under good control at the systematical level.

KEYWords: Phenomenological Models

ArXiv EPrINT: 1911.12883 


\section{Contents}

1 Introduction 1

2 The alternative left-right symmetric model 3

$\begin{array}{lll}3 & \text { Computational setup } & 7\end{array}$

4 Gauge boson mass constraints $\quad 9$

5 Dark matter 12

6 Scotino DM signal at colliders $\quad 16$

$\begin{array}{lll}7 & \text { Summary and conclusions } & 21\end{array}$

$\begin{array}{ll}\text { A Diagonalisation of the scalar sector } & 23\end{array}$

$\begin{array}{ll}\text { B The fermion sector } & 25\end{array}$

C Technical details on our FeynRules implementation 26

\section{Introduction}

The nature of dark matter and its interactions is one of the most puzzling conceptual issues of the Standard Model of particle physics and points clearly towards the existence of new physics. So far, the most popular extensions of the Standard Model (SM) that contain natural dark matter (DM) candidates have been either supersymmetric, so that $R$-parity conservation enforces a stable supersymmetric state behaving as a weakly-interacting massive particle (WIMP) [1], or featuring axion-like particles that could additionally shed light on a potential solution to the strong $C P$ problem $[2,3]$. While experimental DM searches are on-going and put stronger and stronger constraints on the phenomenological viability of the models, several new ad-hoc mechanisms have been recently designed to supplement the SM with a DM candidate. In the latter, the observed properties of DM [4] can be successfully reproduced by an appropriate tuning of the particle masses and properties. For instance, new force carriers could be introduced to mediate the interactions of the dark sector with the SM one, as within the dark photon or vector portal models [5-9]. Differently, the connection between the dark and visible sector could be realised through interactions with vector-like fermions [10-15]. Whilst appealing from a phenomenological point of view by virtue of their simplicity, such DM setups are however quite unnatural. In this work, we therefore go back to natural dark matter models and focus on a less studied class of scenarios that emerges from the grand unification of the SM gauge interactions. 
Grand unification models based on the breaking of the exceptional group $E_{6}[16,17]$ have been popular for awhile, at the beginning as a result of developments in string theories [18], then later as generators of models with additional $U(1)$ symmetries [19]. These so-called $\mathrm{U}(1)^{\prime}$ models arise from considering the $\mathrm{SO}(10) \times \mathrm{U}(1)$ subgroup of $E_{6}$. However, the $E_{6}$ group has also an $\mathrm{SU}(3) \times \mathrm{SU}(3) \times \mathrm{SU}(3)$ subgroup. One of these $\mathrm{SU}(3)$ remains unbroken and is associated with the $\mathrm{SM}$ strong interaction group $\mathrm{SU}(3)_{c}$, while the two others further break into the $\mathrm{SU}(2)_{L} \times \mathrm{SU}(2)_{H} \times \mathrm{U}(1)_{X}$ group that embeds the $\mathrm{SU}(2)_{L} \times \mathrm{U}(1)_{Y}$ electroweak symmetry. In the so-called left-right symmetric model (LRSM), that naturally accounts for non-vanishing neutrino masses [20-23], $\mathrm{SU}(2)_{H}$ is identified with $\mathrm{SU}(2)_{R}$ and $\mathrm{U}(1)_{X}$ with $\mathrm{U}(1)_{B-L}$. In such a configuration, the right-handed SM fermions and the right-handed neutrino $\nu_{R}$ are collected into $\mathrm{SU}(2)_{R}$ doublets. The structure of the Higgs sector could however lead to non-acceptable tree-level flavour-violating interactions that would conflict with the observed properties of kaon and $B$-meson systems. Consequently, the $\mathrm{SU}(2)_{R} \times \mathrm{U}(1)_{B-L}$ symmetry has to be broken at a very high energy scale to masssuppress any potential flavour-violating effect. This additionally pushes the masses of the extra Higgs and gauge bosons of the model to the high scale, making them unlikely to detect at the LHC. Furthermore, in its minimal incarnation, the LRSM lacks any viable DM candidate [24].

It is nevertheless possible to associate the $\mathrm{SU}(2)_{H}$ symmetry with a different $\mathrm{SU}(2)_{R^{\prime}}$ group in which the assignments of the SM fermions into doublets are different $[25,26]$. This model is called the alternative left-right symmetric model (ALRSM) [27, 28]. In this case, the $\mathrm{SU}(2)_{R^{\prime}}$ partner of the right-handed up-quark $u_{R}$ is an exotic down-type quark $d_{R}^{\prime}$ (instead of the SM right-handed down-type quark $d_{R}$ ), and the $\mathrm{SU}(2)_{R^{\prime}}$ partner of the right-handed charged lepton $e_{R}$ is a new neutral lepton, the scotino $n_{R}$ (instead of the more standard right-handed neutrino $\nu_{R}$ ). The right-handed neutrino $\nu_{R}$ and down-type quark $d_{R}$ therefore remain singlets under both the $\mathrm{SU}(2)_{L}$ and $\mathrm{SU}(2)_{R^{\prime}}$ groups. In addition, the model field content also includes $\mathrm{SU}(2)_{L}$ singlet counterparts to the new states, i.e. an $n_{L}$ scotino and a $d_{L}^{\prime}$ down-type quark. Consequently, one generation of quarks is described by one $\mathrm{SU}(2)_{L}$ doublet $Q_{L}=\left(u_{L}, d_{L}\right)$, one $\mathrm{SU}(2)_{R^{\prime}}$ doublet $Q_{R}=\left(u_{R}, d_{R}^{\prime}\right)$ and two $\mathrm{SU}(2)_{L} \times \mathrm{SU}(2)_{R^{\prime}}$ singlets $d_{L}^{\prime}$ and $d_{R}$. Similarly, one generation of leptons is described by one $\mathrm{SU}(2)_{L}$ doublet $L_{L}=\left(\nu_{L}, e_{L}\right)$, one $\mathrm{SU}(2)_{R^{\prime}}$ doublet $L_{R}=\left(n_{R}, e_{R}\right)$ and two $\mathrm{SU}(2)_{L} \times \mathrm{SU}(2)_{R^{\prime}}$ singlets $n_{L}$ and $\nu_{R}$. Moreover, the right-handed neutrino $\nu_{R}$ and the $n_{L}$ scotino being singlets under $\mathrm{U}(1)_{B-L}$, are unlikely to be viable DM candidates, as their too weak interactions with the SM particles would make them over-abundant. On the contrary, the $n_{R}$ scotino may fulfill the role.

In this work, we will show that this is indeed the case. The $n_{R}$ scotino can be an acceptable DM candidate satisfying requirements from imposing agreement with the observed relic density and the non-violation of the DM direct and indirect detection bounds. This however yields very stringent constraints on the model parameter space. In contrast with the usual LRSM, the charged right-handed gauge boson $W^{\prime}$ couples right-handed uptype quarks and charged leptons to their exotic quarks and scotino partners. Therefore, the limits on the $W^{\prime}$-boson mass (originating mainly from the properties of the $K^{0}-\bar{K}^{0}$ mixing in the LRSM case [29]) do not apply. Similarly, the different couplings of the Higgs 
states to fermions forbid most dangerous flavour-violating effects, so that the mass limits on the Higgs states can also be relaxed. As will be demonstrated in the rest of this paper, these considerations lead to a quite predictable lower-energy spectrum with signatures potentially observable at the high-luminosity LHC.

The aim of this work is therefore to provide a comprehensive analysis of the ALRSM setup, emphasising for the first time the complementarity between cosmological, low-energy and collider constraints in this class of extensions of the SM. We update and extent previous recent works that have focused on the dark matter [30] and collider [31] phenomenology independently. In section 2, we provide a brief description of the ALRSM and detail the technical setup underlying our analysis in section 3. Our results are presented in the next sections. In section 4 , we analyse the constraints on the model parameter space originating from LHC searches for new gauge bosons, performed in a similar way as for the LRSM [32]. Section 5 is dedicated to cosmological considerations and their impact on the parameter space. In section 6 we focus on determining promising signals of the model at the future high-luminosity upgrade of the LHC. We summarise our work and conclude in section 7. In appendices $\mathrm{A}$ and $\mathrm{B}$, we include further details on the diagonalisation of the model Higgs and fermionic sector respectively, and document our implementation of the ALRSM in FeynRules [33] in appendix C.

\section{The alternative left-right symmetric model}

The alternative left-right symmetric model [25-28] is a variant of the more usual minimal left-right symmetric model. It is based on the $\mathrm{SU}(3)_{c} \times \mathrm{SU}(2)_{L} \times \mathrm{SU}(2)_{R^{\prime}} \times \mathrm{U}(1)_{B-L}$ gauge group, to which we supplement a global $\mathrm{U}(1)_{S}$ symmetry. The spontaneous breaking of $\mathrm{SU}(2)_{R^{\prime}} \times \mathrm{U}(1)_{S}$ is implemented so that the $L=S+T_{3 R}$ charge, that can be seen as a generalised lepton number, remains unbroken (with $T_{3 R}$ being the third generator of $\left.\mathrm{SU}(2)_{R^{\prime}}\right)$.

The quantum numbers and representations chosen for the fermionic field content of the ALRSM are motivated by heterotic superstring models in which all SM matter multiplets are collected into a 27-plet of $E_{6}$. Under the $E_{6}$ maximal subgroup $\mathrm{SU}(3)_{c} \times \mathrm{SU}(3)_{L} \times$ $\mathrm{SU}(3)_{H}$, the $\mathbf{2 7}$ representation is decomposed as

$$
\mathbf{2 7}=(\mathbf{3}, \mathbf{3}, 1)+(\overline{\mathbf{3}}, 1, \overline{\mathbf{3}})+(1, \overline{\mathbf{3}}, \mathbf{3}) \equiv q+\bar{q}+l .
$$

Explicitly, the particle content for this decomposition can be written, ignoring the sign structure for clarity, as

$$
q=\left(\begin{array}{c}
u_{L} \\
d_{L} \\
d_{L}^{\prime}
\end{array}\right), \quad \bar{q}=\left(\begin{array}{lll}
u_{R}^{c} & d_{R}^{c} & d_{R}^{\prime c}
\end{array}\right), \quad l=\left(\begin{array}{ccc}
E_{R}^{c} & N_{L} & \nu_{L} \\
N_{R}^{c} & E_{L} & e_{L} \\
e_{R}^{c} & \nu_{R}^{c} & n_{R}^{c}
\end{array}\right),
$$

where $d^{\prime}, E, N$ and $n$ are exotic fermions and $u, d, e$ and $\nu$ are the usual up-type quarks, down-type quarks, charged leptons and neutrinos. In this setup, $\mathrm{SU}(3)_{L}$ operates vertically and $\mathrm{SU}(3)_{H}$ horizontally. There are three different ways to embed $\mathrm{SU}(2)_{H}$ into $\mathrm{SU}(3)_{H}[25]$. 


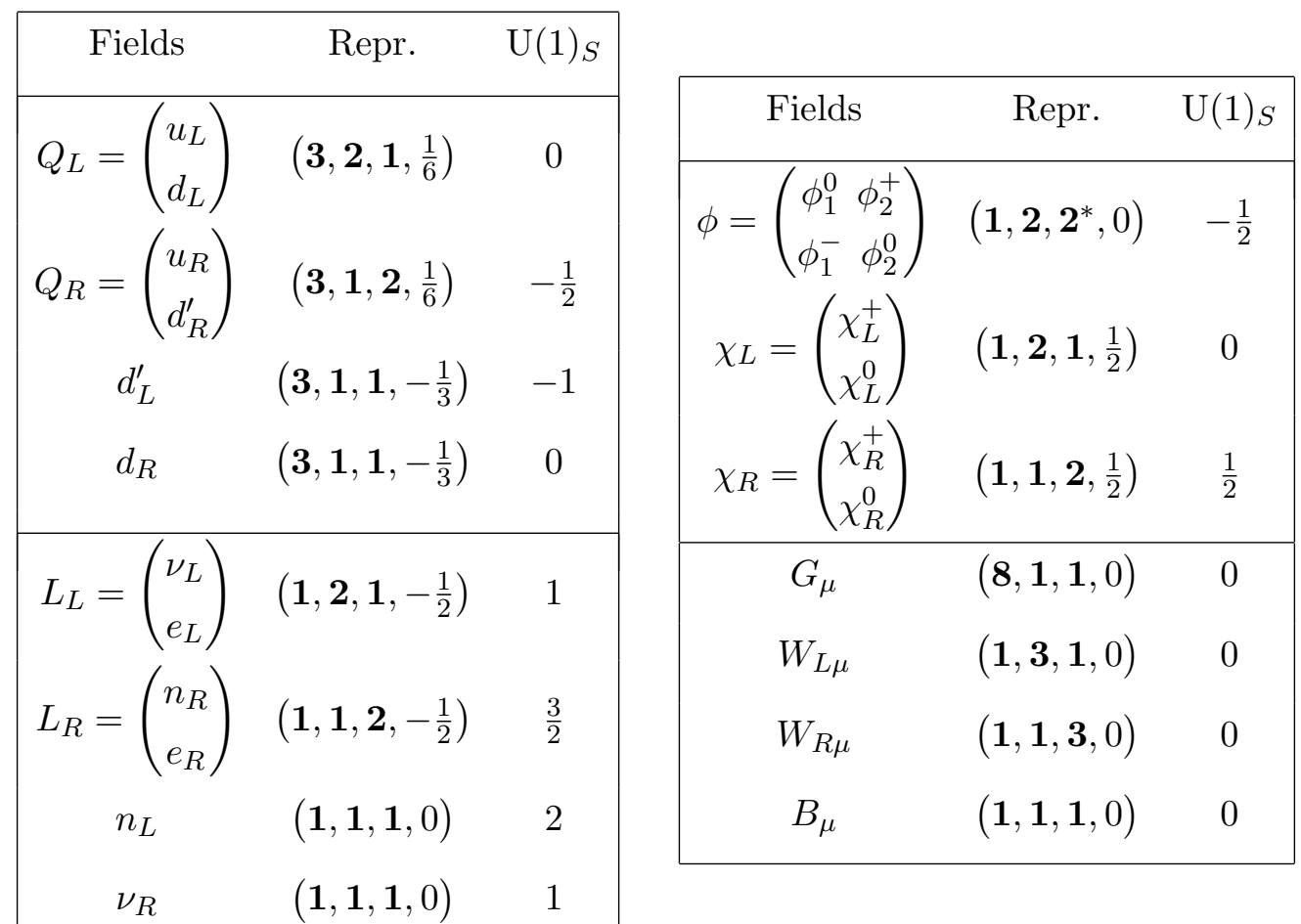

Table 1. ALRSM particle content, given together with the representation of each field under $\mathrm{SU}(3)_{c} \times \mathrm{SU}(2)_{L} \times \mathrm{SU}(2)_{R^{\prime}} \times \mathrm{U}(1)_{B-L}$ (second column) and the $\mathrm{U}(1)_{S}$ quantum numbers (third column). We consider the matter sector (left panel), the gauge sector (lower right panel) and the Higgs sector (upper right panel) separately.

The most common one consists in imposing the first and second column of the above multiplets to form $\mathrm{SU}(2)_{H}$ doublets, which corresponds to the usual LRSM $\left(\mathrm{SU}(2)_{H}=\right.$ $\left.\mathrm{SU}(2)_{R}\right)$ [20-23]. The second option requires in contrast that the first and third columns of the above multiplets form an $\mathrm{SU}(2)_{H}$ doublet, which corresponds to the $\operatorname{ALRSM}\left(\mathrm{SU}(2)_{H}=\right.$ $\left.\mathrm{SU}(2)_{R^{\prime}}\right)[25-28]$. Finally, the third and last option corresponds to doublets formed from the second and third columns of the above multiplets, which corresponds to the Inert Doublet Model $\left(\mathrm{SU}(2)_{H}=\mathrm{SU}(2)_{I}\right)[34-36]$.

We are interested here in the second option. In the rest of this section, we present a summary of the model description, leaving computational details for the appendix. While previous descriptions of the ALRSM exist, we provide extensive details to properly and consistently define our notations, which is relevant for the model implementation in the high-energy physics tools depicted in section 3.

Pairing the fields presented in eq. (2.2) into $\mathrm{SU}(3)_{c} \times \mathrm{SU}(2)_{L} \times \mathrm{SU}(2)_{R^{\prime}} \times \mathrm{U}(1)_{B-L}$ multiplets yields phenomenological issues for the neutrino sector, as the lightest neutrinos get masses of the order of the up quark mass [37]. This can be cured by adding an $E_{6}$ singlet scotino $n_{L}$ to the field content, together with a pair of (heavy) $\mathbf{2 7}+\overline{\mathbf{2 7}}$ Higgs fields. As a consequence, the exotic $E$ and $N$ fermions become much heavier and can be phenomenologically ignored. The resulting fermionic content of the model is presented in the left panel of table 1, together with the representations under the model gauge group 
and the associated $\mathrm{U}(1)_{S}$ quantum numbers. The electric charge of the different fields can be obtained through a generalised Gell-Mann-Nishijima relation $Q=T_{3 R}+T_{3 L}+Y_{B-L}$, which subsequently explains the unconventional $B-L$ charges.

In order to recover the electroweak symmetry group, the gauge and global symmetry $\mathrm{SU}(2)_{R^{\prime}} \times \mathrm{U}(1)_{B-L} \times \mathrm{U}(1)_{S}$ is first broken down to the hypercharge $\mathrm{U}(1)_{Y}$ while preserving the generalised lepton number $L$. This is achieved through an $\mathrm{SU}(2)_{R^{\prime}}$ doublet of scalar fields $\chi_{R}$ charged under $\mathrm{U}(1)_{S}$. While we introduce an $\mathrm{SU}(2)_{L}$ counterpart $\chi_{L}$ to maintain the left-right symmetry, the latter is in contrast blind to the global $\mathrm{U}(1)_{S}$ symmetry. The electroweak symmetry is then broken down to electromagnetism by means of a bidoublet of Higgs fields charged under both $\mathrm{SU}(2)_{L}$ and $\mathrm{SU}(2)_{R^{\prime}}$, but with no $B-L$ quantum numbers. We refer to the right panel of table 1 for details on the gauge and Higgs sector of the ALRSM.

The model Lagrangian includes, on top of standard gauge-invariant kinetic terms for all fields, a Yukawa interaction Lagrangian $\mathcal{L}_{\mathrm{Y}}$ and a scalar potential $V_{\mathrm{H}}$. The most general Yukawa Lagrangian allowed by the gauge and the global $\mathrm{U}(1)_{S}$ symmetries is given by

$$
\begin{aligned}
\mathcal{L}_{\mathrm{Y}}= & \bar{Q}_{L} \hat{\mathbf{Y}}^{u} \hat{\phi}^{\dagger} Q_{R}-\bar{Q}_{L} \hat{\mathbf{Y}}^{d} \chi_{L} d_{R}-\bar{Q}_{R} \hat{\mathbf{Y}}^{d^{\prime}} \chi_{R} d_{L}^{\prime}-\bar{L}_{L} \hat{\mathbf{Y}}^{e} \phi L_{R} \\
& +\bar{L}_{L} \hat{\mathbf{Y}}^{\nu} \hat{\chi}_{L}^{\dagger} \nu_{R}+\bar{L}_{R} \hat{\mathbf{Y}}^{n} \hat{\chi}_{R}^{\dagger} n_{L}+\text { h.c. },
\end{aligned}
$$

where all flavour indices have been omitted for clarity so that the Yukawa couplings $\hat{\mathbf{Y}}$ are $3 \times 3$ matrices in the flavour space, and where the hatted quantities refer to the duals of the scalar fields $\hat{\phi}=\sigma_{2} \phi \sigma_{2}$ and $\hat{\chi}_{L, R}=i \sigma_{2} \chi_{L, R}$ (with $\sigma_{2}$ being the second Pauli matrix). The most general Higgs potential $V_{\mathrm{H}}$ preserving the left-right symmetry is given, following standard conventions [38], by

$$
\begin{aligned}
V_{\mathrm{H}}= & -\mu_{1}^{2} \operatorname{Tr}\left[\phi^{\dagger} \phi\right]-\mu_{2}^{2}\left[\chi_{L}^{\dagger} \chi_{L}+\chi_{R}^{\dagger} \chi_{R}\right]+\lambda_{1}\left(\operatorname{Tr}\left[\phi^{\dagger} \phi\right]\right)^{2}+\lambda_{2}(\phi \cdot \hat{\phi})\left(\hat{\phi}^{\dagger} \cdot \phi^{\dagger}\right) \\
& +\lambda_{3}\left[\left(\chi_{L}^{\dagger} \chi_{L}\right)^{2}+\left(\chi_{R}^{\dagger} \chi_{R}\right)^{2}\right]+2 \lambda_{4}\left(\chi_{L}^{\dagger} \chi_{L}\right)\left(\chi_{R}^{\dagger} \chi_{R}\right) \\
& +2 \alpha_{1} \operatorname{Tr}\left[\phi^{\dagger} \phi\right]\left[\chi_{L}^{\dagger} \chi_{L}+\chi_{R}^{\dagger} \chi_{R}\right]+2 \alpha_{2}\left[\left(\chi_{L}^{\dagger} \phi\right)\left(\chi_{L} \phi^{\dagger}\right)+\left(\phi^{\dagger} \chi_{R}^{\dagger}\right)\left(\phi \chi_{R}\right)\right] \\
& +2 \alpha_{3}\left[\left(\chi_{L}^{\dagger} \hat{\phi}^{\dagger}\right)\left(\chi_{L} \hat{\phi}\right)+\left(\hat{\phi} \chi_{R}^{\dagger}\right)\left(\hat{\phi}^{\dagger} \chi_{R}\right)\right]+\kappa\left[\chi_{L}^{\dagger} \phi \chi_{R}+\chi_{R}^{\dagger} \phi^{\dagger} \chi_{L}\right]
\end{aligned}
$$

and contains bilinear $(\mu)$, trilinear $(\kappa)$ and quartic $(\lambda, \alpha)$ contributions. In the above expression, the dot to the $\mathrm{SU}(2)$-invariant product.

After the breaking of the left-right symmetry down to electromagnetism, the neutral components of the scalar fields acquire non-vanishing vacuum expectation values (vevs),

$$
\langle\phi\rangle=\frac{1}{\sqrt{2}}\left(\begin{array}{ll}
0 & 0 \\
0 & k
\end{array}\right), \quad\left\langle\chi_{L}\right\rangle=\frac{1}{\sqrt{2}}\left(\begin{array}{c}
0 \\
v_{L}
\end{array}\right), \quad\left\langle\chi_{R}\right\rangle=\frac{1}{\sqrt{2}}\left(\begin{array}{c}
0 \\
v_{R}
\end{array}\right),
$$

with the exception of $\phi_{1}^{0}$, which is protected by the conservation of the generalised lepton number that also forbids mixing between the SM $d$ and exotic $d^{\prime}$ quarks. Moreover, all scalar fields with the same electric charge mix. Expressing the complex neutral scalar fields 
in terms of their real degrees of freedom,

$$
\begin{aligned}
\phi_{1}^{0} & =\frac{1}{\sqrt{2}}\left[\Re\left\{\phi_{1}^{0}\right\}+i \Im\left\{\phi_{1}^{0}\right\}\right], \\
\phi_{2}^{0} & =\frac{1}{\sqrt{2}}\left[k+\Re\left\{\phi_{2}^{0}\right\}+i \Im\left\{\phi_{2}^{0}\right\}\right], \\
\chi_{L, R}^{0} & =\frac{1}{\sqrt{2}}\left[v_{L, R}+\Re\left\{\chi_{L, R}^{0}\right\}+i \Im\left\{\chi_{L, R}^{0}\right\}\right],
\end{aligned}
$$

we can write the mixing relations involving the massive $C P$-even Higgs bosons $H_{i}^{0}$ (with $i=0,1,2,3$ ), the massive $C P$-odd Higgs bosons $A_{i}^{0}$ (with $i=1,2$ ) and the two massless Goldstone bosons $G_{1}^{0}$ and $G_{2}^{0}$ that give rise to the longitudinal degrees of freedom of the $Z$ and $Z^{\prime}$ bosons, as

$$
\left(\begin{array}{c}
\Im\left\{\phi_{1}^{0}\right\} \\
\Im\left\{\phi_{2}^{0}\right\} \\
\Im\left\{\chi_{L}^{0}\right\} \\
\Im\left\{\chi_{R}^{0}\right\}
\end{array}\right)=\left(\begin{array}{cccc}
1 & 0 & 0 & 0 \\
0 & & \\
0 & U_{3 \times 3}^{\mathrm{A}} \\
0 &
\end{array}\right)\left(\begin{array}{c}
A_{1}^{0} \\
G_{1}^{0} \\
G_{2}^{0} \\
A_{2}^{0}
\end{array}\right) \text { and }\left(\begin{array}{c}
\Re\left\{\phi_{1}^{0}\right\} \\
\Re\left\{\phi_{2}^{0}\right\} \\
\Re\left\{\chi_{L}^{0}\right\} \\
\Re\left\{\chi_{R}^{0}\right\}
\end{array}\right)=\left(\begin{array}{llll}
1 & 0 & 0 & 0 \\
0 & & \\
0 & U_{3 \times 3}^{\mathrm{H}} \\
0 &
\end{array}\right)\left(\begin{array}{c}
H_{1}^{0} \\
H_{0}^{0} \\
H_{2}^{0} \\
H_{3}^{0}
\end{array}\right) \text {. }
$$

The $\phi_{1}^{0}$ field has been prevented from any mixing by virtue of the conservation of the generalised lepton number, and we refer to appendix A for the expressions of the $3 \times 3$ Higgs mixing matrices $U_{3 \times 3}^{\mathrm{A}}$ and $U_{3 \times 3}^{\mathrm{H}}$, as well as for those of the six Higgs-boson masses. In the charged sector, the $\phi_{1}^{ \pm}, \phi_{2}^{ \pm}, \chi_{L}^{ \pm}$and $\chi_{R}^{ \pm}$fields mix into two physical massive charged Higgs bosons $H_{1}^{ \pm}$and $H_{2}^{ \pm}$, as well as two massless Goldstone bosons $G_{1}^{ \pm}$and $G_{2}^{ \pm}$that are absorbed by the $W$ and $W^{\prime}$ gauge bosons,

$$
\left(\begin{array}{c}
\phi_{2}^{ \pm} \\
\chi_{L}^{ \pm}
\end{array}\right)=\left(\begin{array}{cc}
\cos \beta & \sin \beta \\
-\sin \beta & \cos \beta
\end{array}\right)\left(\begin{array}{c}
H_{1}^{ \pm} \\
G_{1}^{ \pm}
\end{array}\right), \quad\left(\begin{array}{c}
\phi_{1}^{ \pm} \\
\chi_{R}^{ \pm}
\end{array}\right)=\left(\begin{array}{cc}
\cos \zeta & \sin \zeta \\
-\sin \zeta & \cos \zeta
\end{array}\right)\left(\begin{array}{c}
H_{2}^{ \pm} \\
G_{2}^{ \pm}
\end{array}\right)
$$

with

$$
\tan \beta=\frac{k}{v_{L}} \quad \text { and } \quad \tan \zeta=\frac{k}{v_{R}} .
$$

We refer again to appendix A for the explicit expressions of the masses of the physical states in terms of other model parameters.

By definition, the breaking of the left-right symmetry generates masses for the model gauge bosons and induces their mixing (from the Higgs-boson kinetic terms). The charged $W=W_{L}$ and $W^{\prime}=W_{R}$ bosons do not mix as $\left\langle\phi_{1}^{0}\right\rangle=0$, and their masses are given by

$$
M_{W}=\frac{1}{2} g_{L} \sqrt{k^{2}+v_{L}^{2}} \equiv \frac{1}{2} g_{L} v \quad \text { and } \quad M_{W^{\prime}}=\frac{1}{2} g_{R} \sqrt{k^{2}+v_{R}^{2}} \equiv \frac{1}{2} g_{R} v^{\prime} .
$$

In the neutral sector, the gauge boson squared mass matrix is written, in the $\left(B_{\mu}, W_{L \mu}^{3}, W_{R \mu}^{3}\right)$ basis, as

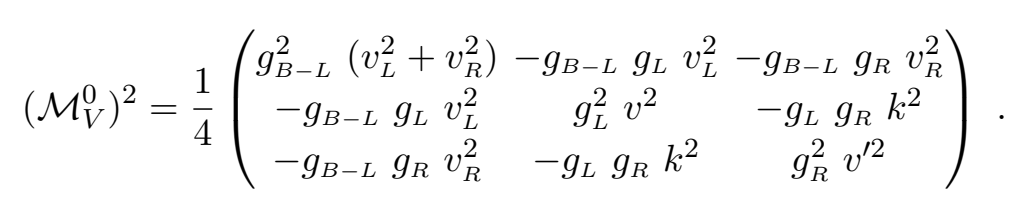


It can be diagonalised through three rotations that mix the $B, W_{L}^{3}$ and $W_{R}^{3}$ bosons into the massless photon $A$ and massive $Z$ and $Z^{\prime}$ states,

$$
\left(\begin{array}{c}
B_{\mu} \\
W_{L \mu}^{3} \\
W_{R \mu}^{3}
\end{array}\right)=\left(\begin{array}{ccc}
c_{\varphi_{W}} & 0 & -s_{\varphi_{W}} \\
0 & 1 & 0 \\
s_{\varphi_{W}} & 0 & c_{\varphi_{W}}
\end{array}\right)\left(\begin{array}{ccc}
c_{\theta_{W}} & -s_{\theta_{W}} & 0 \\
s_{\theta_{W}} & c_{\theta_{W}} & 0 \\
0 & 0 & 1
\end{array}\right)\left(\begin{array}{ccc}
1 & 0 & 0 \\
0 & c_{\vartheta_{W}} & -s_{\vartheta_{W}} \\
0 & s_{\vartheta_{W}} & c_{\vartheta_{W}}
\end{array}\right)\left(\begin{array}{c}
A_{\mu} \\
Z_{\mu} \\
Z_{\mu}^{\prime}
\end{array}\right),
$$

where $s_{i}$ and $c_{i}$ respectively denote the sine and cosine of the angle $i$. The $\varphi_{W}$-rotation mixes the $B$ and $W_{R}^{3}$ bosons into the hypercharge boson $B^{\prime}$ as generated by the breaking of $\mathrm{SU}(2)_{R^{\prime}} \times U_{B-L}$ into to the hypercharge group $\mathrm{U}(1)_{Y}$. The $\theta_{W}$-rotation denotes the usual electroweak mixing, and the $\vartheta_{W}$-rotation is related to the strongly constrained $Z / Z^{\prime}$ mixing. The various mixing angles are defined by

$$
\begin{aligned}
s_{\varphi_{W}} & =\frac{g_{B-L}}{\sqrt{g_{B-L}^{2}+g_{R}^{2}}}=\frac{g_{Y}}{g_{R}} \quad \text { and } \quad s_{\theta_{W}}=\frac{g_{Y}}{\sqrt{g_{L}^{2}+g_{Y}^{2}}}=\frac{e}{g_{L}}, \\
\tan \left(2 \vartheta_{W}\right) & =\frac{2 c_{\varphi_{W}} c_{\theta_{W}} g_{L} g_{R}\left(c_{\varphi_{W}}^{2} k^{2}-s_{\varphi_{W}}^{2} v_{L}^{2}\right)}{-\left(g_{L}^{2}-c_{\varphi_{W}}^{2} c_{\theta_{W}}^{2} g_{R}^{2}\right) c_{\varphi_{W}}^{2} k^{2}-\left(g_{L}^{2}-c_{\theta_{W}}^{2} g_{B-L}^{2} s_{\varphi_{W}}^{2}\right) c_{\varphi_{W}}^{2} v_{L}^{2}+c_{\theta_{W}}^{2} g_{R}^{2} v_{R}^{2}},
\end{aligned}
$$

where $g_{Y}$ and $e$ denote the hypercharge and electromagnetic coupling constant respectively. Neglecting the $Z / Z^{\prime}$ mixing, the $Z$ and $Z^{\prime}$ boson masses are given by

$$
M_{Z}=\frac{g_{L}}{2 c_{\theta_{W}}} v \quad \text { and } \quad M_{Z^{\prime}}=\frac{1}{2} \sqrt{g_{B-L}^{2} s_{\varphi_{W}}^{2} v_{L}^{2}+\frac{g_{R}^{2}\left(c_{\varphi_{W}}^{4} k^{2}+v_{R}^{2}\right)}{c_{\varphi_{W}}^{2}}} .
$$

The breaking of the gauge symmetry furthermore generates masses and mixings in the fermion sector. The masses of the up-type quark and charged leptons are controlled by the vev $k$ of the Higgs bidoublet, whereas the masses of the neutrinos and the down-type quarks arise from the vev $v_{L}$ of the $\chi_{L}$ Higgs triplet. The scale of the exotic fermion masses is in contrast solely induced by the vev $v_{R}$ of the $\chi_{R}$ triplet. Similarly to what is achieved in the LRSM, all fermion mixing are conveniently absorbed into two CKM ( $V_{\mathrm{CKM}}$ and $\left.V_{\mathrm{CKM}^{\prime}}\right)$ and two PMNS ( $V_{\mathrm{PMNS}}$ and $\left.V_{\mathrm{PMNS}^{\prime}}\right)$ rotations,

$$
d_{L} \rightarrow V_{\mathrm{CKM}} d_{L}, \quad \nu_{L} \rightarrow V_{\mathrm{PMNS}} d_{L}, \quad d_{R}^{\prime} \rightarrow V_{\mathrm{CKM}^{\prime}} d_{R}^{\prime}, \quad n_{R} \rightarrow V_{\mathrm{PMNS}^{\prime}} n_{R} .
$$

We refer to appendix B for additional details on the generation of the fermion masses, and their explicit expression in terms of the other model free parameters.

Finally, we supplement the model Lagrangian by the effective couplings $a_{\mathrm{H}}^{g}$ and $a_{\mathrm{H}}^{a}$ of the SM Higgs boson to gluons and photons,

$$
\mathcal{L}_{\mathrm{eff}}=-\frac{1}{4} a_{\mathrm{H}}^{g} H_{0}^{0} G_{\mu \nu}^{a} G_{a}^{\mu \nu}-\frac{1}{4} a_{\mathrm{H}}^{a} H_{0}^{0} F_{\mu \nu} F^{\mu \nu},
$$

where $G_{\mu \nu}^{a}$ and $F_{\mu \nu}$ respectively denote the gluon and photon field strength tensors.

\section{Computational setup}

To perform our analysis of the cosmology and collider phenomenology of the ALRSM, we have implemented the model presented in section 2 into FeynRuLEs (version 2.3.35) [33]. 
Whereas an implementation was already publicly available for many years [31, 39], we found several issues with the latter that justified the development of a new implementation from scratch. First, the Goldstone sector is incorrectly implemented in the existing implementation, which could yield wrong predictions when jointly used with a tool handling computations in Feynman gauge by default (like MicrOMEGAs [40]). Secondly, all scalar fields are doubly-declared (i.e. both under their standard and dual form), the implementation is only partly relying on FEYNRULES built-in functions to treat index contractions and covariant derivatives, and the declaration of the model parameters relies particularly heavily on the existence of an unnecessary large amount of temporary intermediate abbreviations. This consequently renders the implementation hard to verify and understand. Moreover, the electroweak sector is defined by five independent parameters instead of three. Thirdly, the existing implementation enforces the unnecessary equality $g_{L}=g_{R}$, that is justified neither theoretically nor phenomenologically. Relaxing this constraint would have required to modify all relations relevant for the gauge and Higgs boson masses and mixings (see section 2 and appendix A), which would have been quite a complex task given the heavy handling of the model parameters. Finally, the original implementation has also the $V_{\mathrm{CKM}}=V_{\mathrm{CKM}^{\prime}}$ and $V_{\mathrm{PMNS}}=V_{\mathrm{PMNS}^{\prime}}$ equalities built in, which is again not justified (see appendix B). For all those reasons, we decided on designing a fresh, more general, implementation, that is also publicly released on the FEYNRULES model database. ${ }^{1}$ In order to facilitate the usage of our FEYNRULES implementation, we document it further in appendix C, where we provide information on the new physics mass-eigenstates supplementing the SM field content, the free model parameters and their relation to all the other (internal) parameters.

We have then made use of FeynRules to generate CAlcHep [41] model files and a UFO [42] version of the model [43], so that we could employ MicrOMEGAs (version 5.0.8) [40] for the computation of the predictions relevant for our dark matter study, and MG5_AMC (version 2.6.4) [44] for generating the hard-scattering event samples necessary for our collider study. These events, obtained by convoluting the hard-scattering matrix elements with the leading-order set of NNPDF 2.3 parton densities [45], are subsequently matched with the PYтнIA 8 (version 8.243) [46] parton showering and hadronisation algorithms, and we simulate the typical response of an LHC detector by means of the Delphes 3 [47] programme (version 3.4.2) that internally relies on the anti- $k_{T}$ algorithm [48] as implemented into FASTJET [49] (version 3.3.2) for event reconstruction. We have employed MADANALYsis 5 [50] (version 1.8.23) for the collider analysis of section 6. Moreover, we have additionally used the generated UFO model with MADDM [51] to independently verify the results obtained with MicrOMEGAs, in particular for what concerns gauge invariance.

In addition, we have relied on HiggsBounds (version 4.3.1) [52] and HiggsSignals (version 1.4.0) [53] to verify the compatibility of the ALRSM Higgs sector with data, with the $H_{0}^{0}$ field being associated with the SM Higgs boson. We have used the PySLHA package [54] to read the input values for the model parameters that we encode under

\footnotetext{
${ }^{1}$ See http://feynrules.irmp.ucl.ac.be/wiki/ALRM_general.
} 


\begin{tabular}{|cc|cc|}
\hline Parameter & Scanned range & Parameter & Scanned range \\
\hline $\tan \beta$ & {$[0.7,50]$} & $m_{n_{1}}$ & {$[10,2000] \mathrm{GeV}$} \\
$g_{R}$ & {$[0.37,0.768]$} & $m_{n_{2}}$ & {$[10,2000] \mathrm{GeV}$} \\
$v^{\prime}$ & {$[6.5,13] \mathrm{TeV}$} & $m_{n_{3}}$ & {$[10,2000] \mathrm{GeV}$} \\
\hline$\lambda_{2}$ & 0. & $m_{d^{\prime}}$ & {$[500,2000] \mathrm{GeV}$} \\
$\lambda_{3}$ & {$[0.01,0.09]$} & $m_{s^{\prime}}$ & {$\left[m_{d^{\prime}}, 2500\right] \mathrm{GeV}$} \\
$\kappa$ & {$[-50,-1] \mathrm{GeV}$} & $m_{b^{\prime}}$ & {$\left[m_{s^{\prime}}, 3000\right] \mathrm{GeV}$} \\
$\alpha_{1}=\alpha_{2}=\alpha_{3}$ & {$[0.01,0.5]$} & & \\
\hline
\end{tabular}

Table 2. Ranges where the new parameters defining the new physics sector of the model are allowed to vary.

the SLHA format [55], and to integrate the various employed programmes into a single framework. Using our interfacing, we performed a random scan of the model parameter space following the Metropolis-Hastings technique. We have fixed the SM parameters to their Particle Data Group (PDG) values [56], chosen the $V_{\mathrm{CKM}^{\prime}}$ and $V_{\mathrm{PMNS}^{\prime}}$ matrices to be equal to their SM counterparts, and varied the remaining 15 parameters as described in table 2 .

The $\mathrm{SU}(2)_{R^{\prime}}$ coupling $g_{R}$ is allowed to vary within the $[0.37,0.768]$ window. The lower bound originates from the $g_{R} / g_{L}$ ratio that is theoretically constrained to be larger than $\tan \theta_{W}$ [57], whereas the upper bound is phenomenological. In practice, $g_{R}$ can indeed vary all up to the perturbative limit of $g_{R}=\sqrt{4 \pi}$. However, imposing an upper bound on $g_{R}$ that is $4-5$ times smaller guarantees scenarios that are viable with respect to LHC limits [58-61] and that feature at least one light extra gauge boson (see section 4). The same light-spectrum considerations has lead to our choices for the values of the $\tan \beta$ and $v^{\prime}$ parameters, with the additional constrains stemming from the expectation that the $\mathrm{SU}(2)_{R^{\prime}}$ symmetry has to be broken in the multi-TeV regime and that the $Z / Z^{\prime}$ mixing must be negligibly small.

The ranges and configuration adopted for the parameters of the Higgs sector are driven by the Higgs potential minimisation conditions of eqs. (A.2) and (A.3), as well as by the above-mentioned LHC constraints on the $Z^{\prime}$-boson mass, and by the requirement that the lightest charged Higgs boson is not tachyonic. It turns out that all phenomenologically acceptable scenarios feature $\alpha_{1} \sim \alpha_{2}=\alpha_{3}$ and $\lambda_{2}=0$, so that we set for simplicity

$$
\lambda_{2}=0 \quad \text { and } \quad \alpha_{1}=\alpha_{2}=\alpha_{3} .
$$

Moreover, $\lambda_{3}$ has to be small and we recall that $\kappa$ has to be negative (see appendix A). Finally, the exotic quarks and scotino masses are not restricted and we allow them to vary mostly freely, with a phenomenological upper bound allowing them to be not too heavy.

\section{Gauge boson mass constraints}

Following the methodolgy described in the previous section, we scan the parameter space imposing constraints on the properties of the Higgs sector so that the $H_{0}^{0}$ scalar boson is 

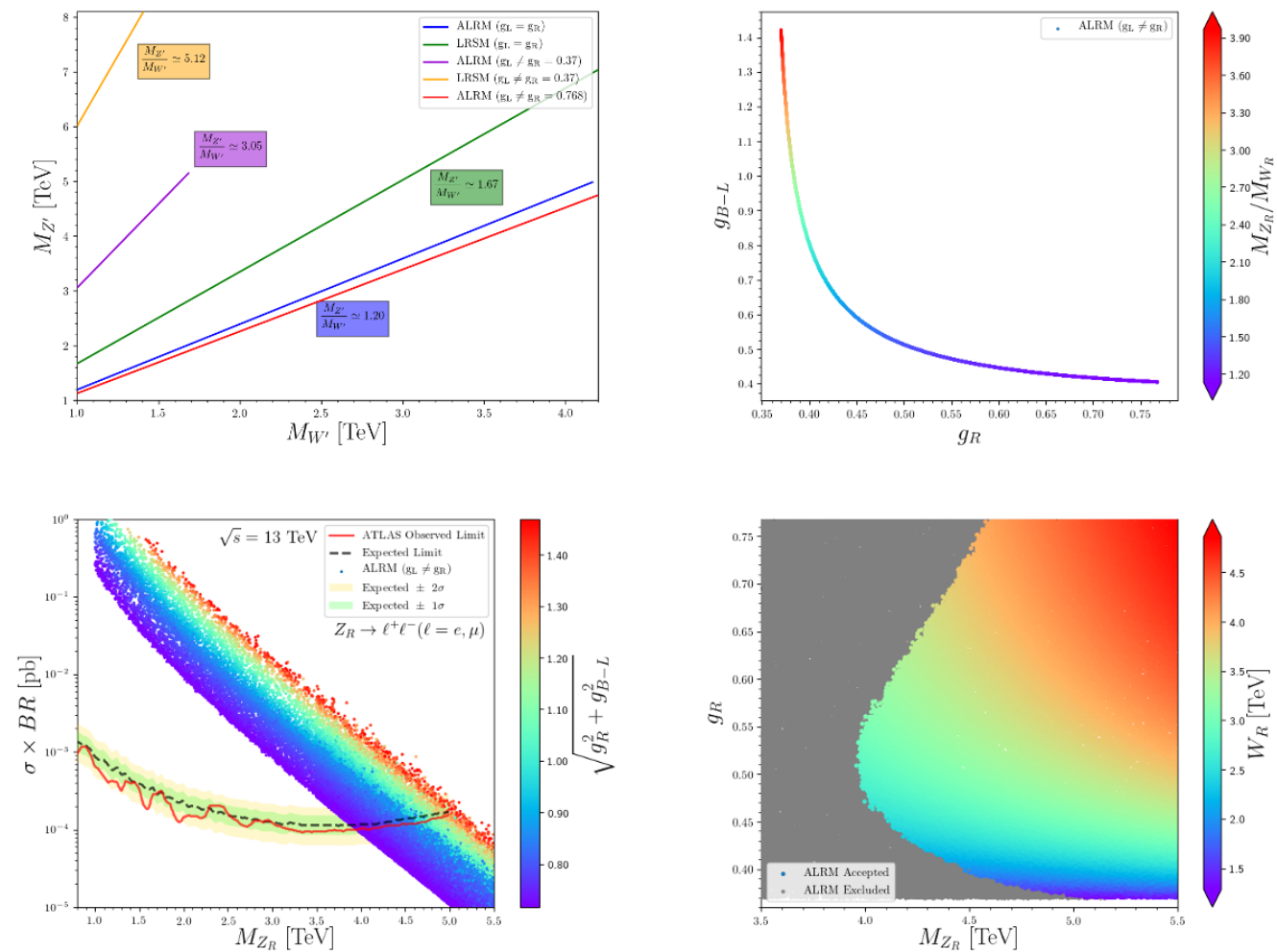

Figure 1. Properties of the gauge sector for the ALRSM scenarios featuring a Higgs sector compatible with data. We emphasise the relations between the $W^{\prime}$ and $Z^{\prime}$ boson masses with the gauge couplings and also investigate the LHC constraints on the mass of the $Z^{\prime}$ boson.

SM-like and has features agreeing with experimental data. In this section, we analyse the properties of the gauge sector for all scenarios accepted in our scanning procedure.

In the upper left and right panels of figure 1, we depict the relations between the masses of the extra gauge bosons $M_{Z^{\prime}}$ and $M_{W^{\prime}}$ and the ALRSM coupling constants $g_{L}$, $g_{B-L}$ and $g_{R}$. We observe, in the upper left panel of the figure, that in the ALRSM the ratio of the neutral to the charged extra boson masses ranges from about 1.20 for a maximal $g_{R}$ value of 0.768 (light green line) to about 3.05 for a minimal setup defined by $g_{R}=0.37$ (purple line). The left-right symmetric case $g_{L}=g_{R} \approx 0.64$ is also indicated (dark blue line). This shows that a large variety of splittings can be realised for gauge boson masses lying in the 1-5 TeV range. Equivalently, both compressed spectra in which the $Z^{\prime}$-boson is only $20 \%$ heavier than the $W^{\prime}$-boson and more split spectra in which the $Z^{\prime}$-boson is more than about 3 times heavier than the $W^{\prime}$-boson are allowed by Higgs data, and this for a large set of $W^{\prime}$-boson masses lying in the $1-4 \mathrm{TeV}$ range. We compare those findings with predictions relevant for the usual LRSM for similar $g_{R}$ values (dark green and orange lines for $g_{L}=g_{R}$ and $g_{R}=0.37$ respectively). It turns out that the $M_{Z^{\prime}} / M_{W^{\prime}}$ ratio is lower in the ALRSM than in the LRSM for a given $g_{R}$ value, i.e. the ALRSM gauge boson spectrum is more compressed than in the standard LRSM for a given $\mathrm{SU}(2)_{R}$ coupling constant value. In the upper right panel of figure 1 , we study the dependence of this mass 
ratio on the $g_{B-L}$ an $g_{R}$ coupling constants. The latter two couplings are related to the hypercharge coupling,

$$
\frac{1}{g_{Y}^{2}}=\frac{1}{g_{R}^{2}}+\frac{1}{g_{B-L}^{2}}
$$

so that large $g_{R}$ values are always associated with low $g_{B-L}$ values and vice versa. In typical scenarios, the hierarchy $v_{L} \ll k \ll v_{R}$ is fulfilled as $v_{L}$ is small (which is also favoured by constraints originating from the $\rho$ parameter [62]), $k$ drives the electroweak vacuum and is of $\mathcal{O}(100) \mathrm{GeV}$, and $v_{R}$ is related to the breaking of the $\mathrm{SU}(2)_{R^{\prime}}$ symmetry and is thus larger. Therefore, eqs. (2.10), (2.13) and (2.14) yield

$$
\frac{M_{Z^{\prime}}}{M_{W^{\prime}}} \approx \frac{1}{c_{\varphi_{W}}}=\frac{g_{B-L}}{g_{Y}}
$$

When $g_{R}$ is larger, $g_{B-L}$ is smaller and $c_{\varphi_{W}}$ is consequently larger. Smaller $M_{Z^{\prime}} / M_{W^{\prime}}$ ratios are thus expected. Conversely, with increasing values of $g_{B-L}, c_{\varphi_{W}}$ and $g_{R}$ become smaller so that the $M_{Z^{\prime}} / M_{W^{\prime}}$ ratio increases. In those case, the $W^{\prime}$ boson can become up to about three times lighter than the $Z^{\prime}$-boson (see the upper left panel of the figure). This feature has profound consequences on the possible existence of light ALRSM $W^{\prime}$ bosons allowed by data.

The $W^{\prime}$-boson does not indeed couple to pairs of ordinary SM fermions, but instead couples to a SM up-type quark and an exotic down-type quark $d^{\prime}$, or an electron and a scotino. It can consequently not be directly produced at colliders and all LHC bounds on an additional $W^{\prime}$ boson originating from dijet and dileptonic resonance searches are automatically evaded [58-61]. Only the neutral ALRSM $Z^{\prime}$-boson can potentially be searched for through standard extra gauge boson LHC analyses, as it is allowed to couple to pairs of SM fermions. We evaluate the resulting bounds in the lower left panel of figure 1 in which we consider the most constraining limits originating from the cleaner searches in the dilepton mode. For each benchmark scenario selected by our scanning procedure, we evaluate the $Z^{\prime}$-boson production cross section, including the branching ratio associated with a $Z^{\prime} \rightarrow e^{+} e^{-}$or $\mu^{+} \mu^{-}$decay, and compare our predictions to the bounds arising from the ATLAS search of ref. [60]. The spread in cross section obtained for a given $Z^{\prime}$ mass stems from the different values of the strength of the $Z^{\prime}$-boson fermionic couplings, which we estimate by $\sqrt{g_{R}^{2}+g_{B-L}^{2}}$ and which is represented through the colour map in the figure. For the smallest coupling values, $Z^{\prime}$ bosons as light as $4 \mathrm{TeV}$ are allowed by data, whilst when the coupling strength gets larger, the limits can be pushed up to $5 \mathrm{TeV} .^{2}$

As previously mentioned and visible from the upper left panel of figure 1 , the $W^{\prime}$ - and $Z^{\prime}$-bosons can feature a very split spectrum so that a $4-5 \mathrm{TeV} Z^{\prime}$ boson can coexist with a $1-2 \mathrm{TeV} W^{\prime}$-boson. This feature is illustrated in the lower right panel of the figure in which we present, for each scenario satisfying the LHC $Z^{\prime}$ bounds (the excluded benchmarks being shown in grey), the corresponding value of the $g_{R}$ coupling. The latter dictates the $W^{\prime}$-boson mass value, as given by eq. (2.10) which we also represent through the colour

\footnotetext{
${ }^{2}$ Whilst in the large coupling case, the $Z^{\prime}$ width over mass ratio can reach $10 \%$, we have verified that our approximation in which we neglect the interferences of the signal with the SM dilepton continuum was reasonably satisfactory.
} 
map. For the lowest $g_{R}$ values allowed in the scan, the additional gauge boson splitting is expected to be the largest (see the upper left panel of figure 1), so that viable scenarios featuring a $W^{\prime}$ boson as light as $1-2 \mathrm{TeV}$ and a $Z^{\prime}$-boson not excluded by present searches are found. The considered $Z^{\prime}$ bounds are expected to slightly improve by about $20 \%$ during the high-luminosity operation phase of the LHC [63], which does not challenge the existence of light $W^{\prime}$ bosons (see the lower right panel of figure 1). The lightest options for the $W^{\prime}$ boson correspond to scenarios featuring the smallest $g_{R}$ value theoretically allowed $\left(g_{R} \sim 0.37\right)$, the $Z^{\prime}$-boson being in this case constrained to lie above roughly $5 \mathrm{TeV}$. Viable scenarios in which the $Z^{\prime}$-boson is lighter, with $M_{Z^{\prime}} \approx 4 \mathrm{TeV}$, are also allowed by data. In that configuration, the $\mathrm{U}(1)_{B-L}$ and $\mathrm{SU}(2)_{R^{\prime}}$ coupling constant are of a similar magnitude, $g_{R} \approx g_{B-L} \sim 0.5$ (see the upper right panel of figure 1 ), and the $W^{\prime} / Z^{\prime}$ boson splitting is smaller $\left(M_{W^{\prime}} \approx 3 \mathrm{TeV}\right)$. Our results also show that the largest $g_{R}$ values correspond to the heaviest scenarios, being thus disfavoured to be observed at current colliders. This motivates the upper bound set on $g_{R}$ in our scan (see section 3 ).

\section{Dark matter}

In this section, we investigate the constraints on the model arising from imposing the lightest scotino as a viable DM candidate with properties compatible with current cosmological data. First, we require that the predicted relic density agrees within $20 \%$ (to conservatively allow for uncertainties on the predictions) with the recent Planck results, $\Omega_{\mathrm{DM}} h^{2}=0.12$ [64]. We calculate, for all points returned by our scanning procedure that are in addition compatible with the LHC $Z^{\prime}$-boson bounds (see section 4), the associated DM relic density. We present our results in figure 2. In all the subfigures, the relic density is given as a function of the mass of the lightest scotino that we denote by $m_{n_{\mathrm{DM}}}$. Two classes of solutions emerge from the results. In a first set of allowed masses, the lightest scotino is quite light, with a mass lying in the $[700,1050] \mathrm{GeV}$ window. The relic density as observed by the Planck collaboration can however also be accommodated when the spectrum is heavier, i.e. with a lightest scotino featuring $m_{n_{\mathrm{DM}}} \in[1.7,2] \mathrm{TeV}$. This last case is naturally less appealing from a collider search point of view. For this reason, we did not increase the scanned scotino mass range (see section 3), although potentially viable scenarios could be obtained for even heavier scotinos, and we mostly ignore this regime in the following discussion. In this case, the right value obtained for the relic density prediction stems from enhanced annihilations into fermions through $Z^{\prime}$-boson $s$-channel exchanges (see the lower right panel of the figure).

In the different panels of figure 2, we analyse the properties of those ALRSM scenarios for which a relic density compatible with Planck data has been found. A first remarkable feature is that when the DM scotino state is light (i.e. when $m_{n_{\mathrm{DM}}} \in[700,1050] \mathrm{GeV}$ ), several Higgs bosons are also light (upper left panel of the figure). The degenerate $H_{1}^{0}$ and $A_{1}^{0}$ neutral states, as well as the charged $H_{2}^{ \pm}$boson, hence have masses of $100-200 \mathrm{GeV}$. The heavier the lightest scotino, the lighter these scalar and pseudoscalar bosons turn out to be. More precisely, for a scotino mass of about $750 \mathrm{GeV}$, the (pseudo)scalar masses are about $200 \mathrm{GeV}$, whilst for a scotino mass of $800-1000 \mathrm{GeV}$, they turn out to be about 

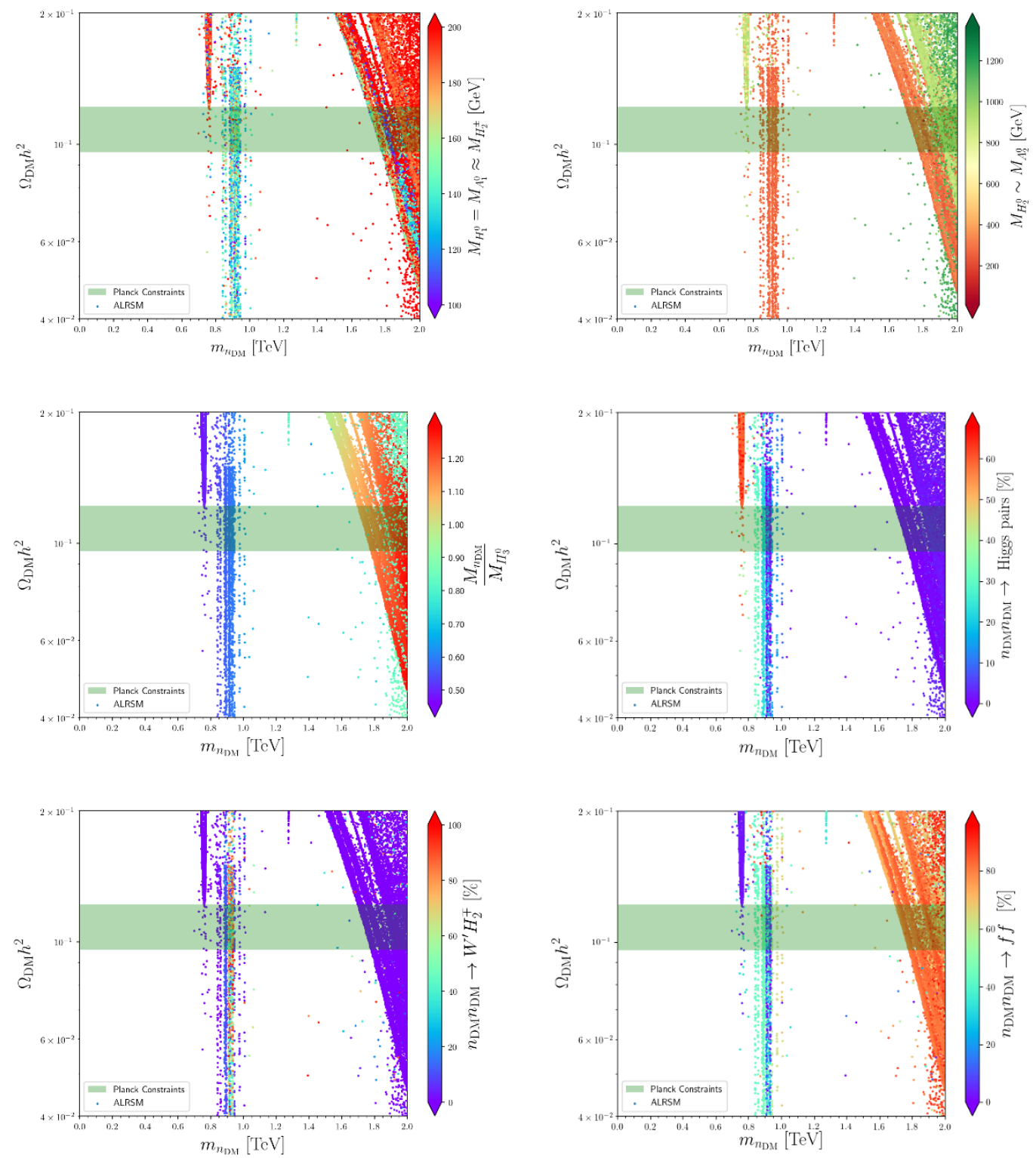

Figure 2. Relic density predictions for all ALRSM scenarios satisfying the Higgs constraints imposed during our scan and compatible with LHC $Z^{\prime}$ bounds, and its dependence on the mass of the lightest scotino. In each panel of the figure, we depict a specific property of all those scenarios. In the upper left panel, we represent by a colour code the mass of the $H_{1}^{0}, A_{1}^{0}$ and $H_{2}^{ \pm}$Higgs states, whilst in the upper right panel, we focus on the one of the $H_{2}^{0}$ and $A_{2}^{0}$ Higgs bosons. The mass of the scalar Higgs boson $H_{3}^{0}$ is presented relatively to the scotino mass in the central left panel, and the fractions of the DM annihilation cross section associated with annihilations in Higgs bosons, $W^{\prime \pm} H_{2}^{\mp}$ systems and fermions pairs are given in the central right, lower left and lower right panels respectively.

$100 \mathrm{GeV}$. Moreover, the second scalar states $H_{2}^{0}$ and $A_{2}^{0}$ are only slightly heavier (upper right panel of figure 2), with masses found to lie around $400 \mathrm{GeV}$. As a consequence of the 


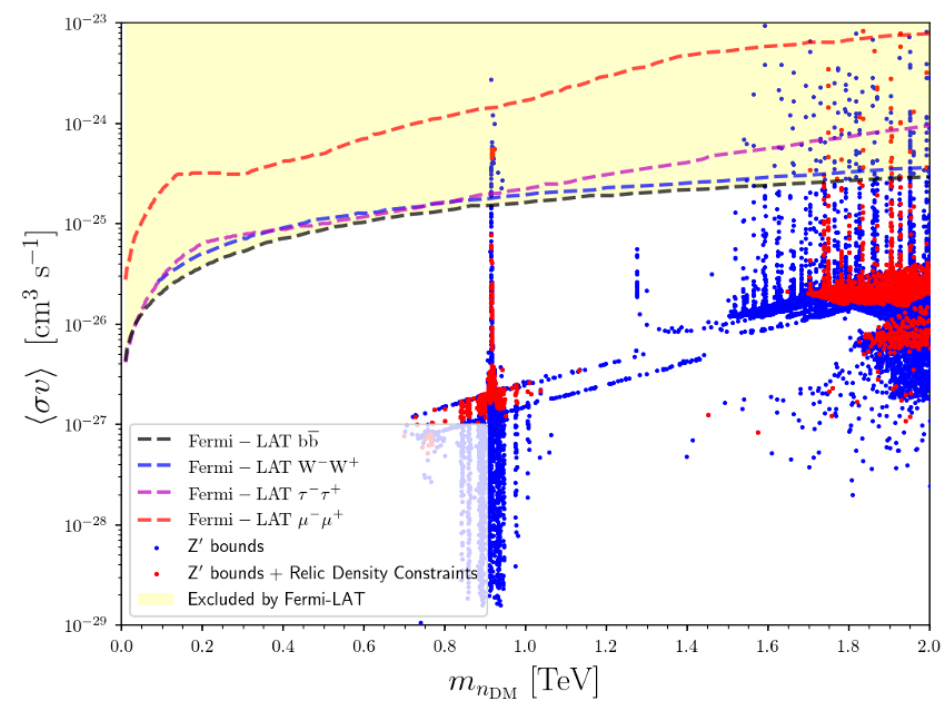

Figure 3. Predictions for the total DM annihilation cross section as a function of the mass of the lightest scotino. We show all points returned by the scan and that are compatible with LHC $Z^{\prime}$ bounds. Scenarios for which the predictions for the relic density agree with Planck data are shown in red, whilst scenarios for which DM is over-abundant or under-abundant are shown in blue. We superimpose to our predictions constraints from Fermi-LAT [65], the yellow area being excluded.

presence of all those light states, scotino annihilations into pairs of Higgs bosons contribute significantly to the total annihilation cross section, as illustrated in the central right panel of figure 2. This figure shows that on the contrary to any other regime probed in our scan, channels where DM annihilates into Higgs bosons contribute about $30-65 \%$ to the total relic density when $m_{n_{\mathrm{DM}}} \in[700,1050] \mathrm{GeV}$. Such an enhancement (by comparison with heavier DM scenarios where those channels are usually negligible) arises from the heaviest scalar state $H_{3}^{0}$ that can mediate several DM annihilation modes. This scalar boson is found to have a mass roughly equal to twice the DM mass $M_{H_{3}^{0}} \approx 2 m_{n_{\mathrm{DM}}}$ (see the central left panel of figure 2). There hence exists a new funnel allowing for efficient DM annihilations into Higgs bosons, preventing DM from being over-abundant. In addition, the $H_{3}^{0}$ funnel also mediates annihilations into $W^{\prime \mp} H_{2}^{ \pm}$systems, that turn to be dominant for a DM mass of about $900 \mathrm{GeV}$ (lower left panel of figure 2).

Whilst we have demonstrated that the lightest scotino could be a viable DM candidate from the point of view of the relic density, it is important to verify that dark matter indirect and direct detection bounds are at the same time satisfied. In figure 3 , we present the value of the total DM annihilation cross section at zero velocity as a function of the scotino mass for all scanned scenarios satisfying the $Z^{\prime}$-boson LHC limits. Configurations for which the relic density is found in agreement with Planck data are shown in red, whilst any other setup returned by the scan is shown in blue. In our predictions, we have moreover rescaled the DM annihilation cross section to its present-day density. We compare our predictions to the latest bounds derived from the Fermi satellite mission data [65]. We depict, as a yellow area, the parameter space region that is found out to be excluded. Most scanned 

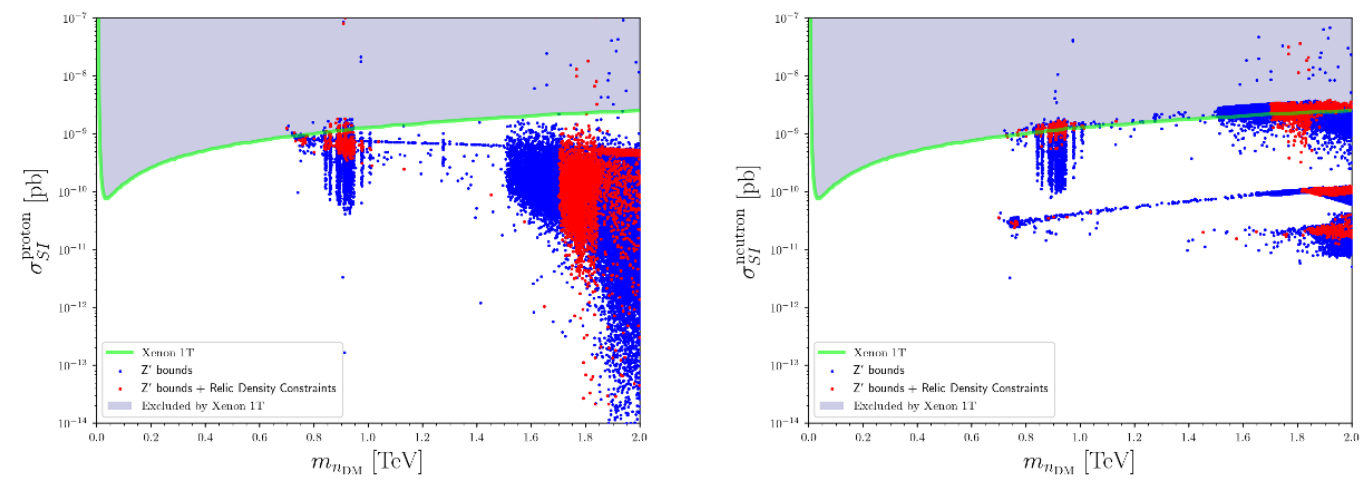

Figure 4. DM-proton (left) and DM-neutron (right) spin-independent scattering cross section as a function of the mass of the lightest scotino $m_{n_{\mathrm{DM}}}$. Red points represent the scenarios featuring a relic density consistent with Planck data, and blue point any other scenario returned by the scan. We restrict the results to scenarios satisfying the LHC $Z^{\prime}$ bounds.

scenarios naturally feature an annihilation cross section that is 1 or 2 orders of magnitude too small to leave any potentially visible signals in Fermi-LAT data, with a few exceptions where the annihilation cross section at present time is enhanced. In general, such an enhancement simultaneously leads to a reduction of the relic density so that Planck data is at the same time accommodated. Equivalently, a significant fraction of the scenarios that are excluded by indirect detection bounds turn out to feature a relic density agreeing with cosmological data (the red points lying within the yellow contour). Fortunately, most potentially viable parameter regions from the relic density standpoint are unaffected by current indirect detection limits and will potentially stay so for some time by virtue of their correspondingly small annihilation cross sections.

In figure 4, we focus on DM direct detection bounds and represent the DM-proton (left panel) and DM-neutron (right panel) spin-independent scattering cross section $\sigma_{\mathrm{SI}}^{\text {proton }}$ and $\sigma_{\mathrm{SI}}^{\text {neutron }}$ as a function of the of the mass of the lightest scotino. Once again, our results are normalised to the present-day relic density and points compatible (incompatible) with Plank data are shown in red (blue). Our predictions are then compared with the results of the Xenon 1T experiment [66]. In the ALRSM, neutron-scotino scattering cross sections are naturally larger than proton-scotino scattering ones by virtue of the differences between the $Z$ and $Z^{\prime}$ couplings to the up-type and down-type quarks, so that stronger constraints arise from the former process. Moreover, the distribution of points in three clusters, as visible in the right panel of figure 4, stem from two features. First, these clusters are associated with different $Z^{\prime}$ mass ranges, lighter $Z^{\prime}$-bosons being associated with smaller neutron-DM scattering rates. Second, down-type quarks play a special role in the ALRSM as they do not couple to the $Z^{\prime}$-boson. This impacts the DM-neutron scattering cross section (consequently due to the larger down-quark content of the neutron) whilst leading to a more 'continuous' behaviour for the DM-proton scattering cross section. A large fraction of all scenarios accommodating the correct relic density are consequently excluded by the Xenon 1T limits on the neutron-DM scattering cross section. Few options featuring 


\begin{tabular}{|c|cccccc|}
\hline & $\tan \beta$ & $g_{R}$ & $v^{\prime}[\mathrm{GeV}]$ & $\lambda_{3}$ & $\kappa[\mathrm{GeV}]$ & $\alpha_{1}=\alpha_{2}=\alpha_{3}$ \\
\hline BM I & 4.58 & 0.374 & 7799 & 0.0196 & -31.08 & 0.0144 \\
BM II & 1.78 & 0.370 & 6963 & 0.0237 & -2.43 & 0.110 \\
BM III & 4.55 & 0.374 & 7799 & 0.0196 & -30.38 & 0.0144 \\
\hline
\end{tabular}

\begin{tabular}{|c|ccccccc|}
\hline$[\mathrm{GeV}]$ & $M_{H_{1}^{0}}$ & $M_{H_{2}^{0}}$ & $M_{H_{3}^{0}}$ & $M_{A_{1}^{0}}$ & $M_{A_{2}^{0}}$ & $M_{H_{1}^{ \pm}}$ & $M_{H_{2}^{ \pm}}$ \\
\hline BM I & 193 & 907 & 1546 & 193 & 907 & 907 & 194 \\
BM II & 82 & 213 & 1578 & 82 & 167 & 167 & 82 \\
BM III & 192 & 894 & 1546 & 192 & 894 & 894 & 192 \\
\hline
\end{tabular}

\begin{tabular}{|c|cccccccc|}
\hline$[\mathrm{GeV}]$ & $M_{Z^{\prime}}$ & $M_{W^{\prime}}$ & $M_{n_{1}}$ & $M_{n_{2}}$ & $M_{n_{3}}$ & $M_{d^{\prime}}$ & $M_{s^{\prime}}$ & $M_{b^{\prime}}$ \\
\hline BM I & 4992 & 1460 & 756 & 971 & 1202 & 1500 & 1800 & 2000 \\
BM II & 5113 & 1288 & 909 & 1134 & 1223 & 1400 & 1822 & 2200 \\
BM III & 4992 & 1460 & 902 & 1023 & 1312 & 1500 & 1936 & 2821 \\
\hline
\end{tabular}

Table 3. Values of the free ALRSM parameters defining our three benchmark scenarios BM I, BM II and BM III (upper panel) and resulting mass spectrum (middle and lower panels). All masses are given in $\mathrm{GeV}$.

a scotino mass in the $700-1050 \mathrm{GeV}$ range survive, made possible by a suppression of the $Z^{\prime}$-boson exchange diagrams due to a larger $Z^{\prime}$ boson mass in those scenarios.

In conclusion, we were able to obtain scenarios satisfying DM relic density and direct and indirect detection constraints. The existence of those scenarios is however pretty constrained, in particular due to direct detection bounds that put severe requirements on the model spectrum, rendering it very predictable. In the surviving scenarios, the lightest scotino (i.e. our DM candidate) has a mass in the $750-1000 \mathrm{GeV}$ window and a set of non-SM-like Higgs bosons are light. In particular, the lightest $H_{1}^{0}$ and $A_{1}^{0}$ bosons, as well as the $H_{2}^{ \pm}$boson, have masses in the $100-200 \mathrm{GeV}$ window. Moreover, the next scalar state $H_{2}^{0}$ and pseudoscalar state $A_{2}^{0}$ are only mildly heavier, with masses in general around $400 \mathrm{GeV}$. The heaviest scalar $H_{3}^{0}$ is in contrast much heavier, with a mass roughly equal to twice the lightest scotino mass. As a consequence of the presence of the funnel topology, the DM annihilation cross section is predicted to be in the right range of values to accommodate Planck data. A small fraction of scenarios are moreover compatible with DM direct and indirect detection bounds. Another general feature is that those scenarios feature a potentially light $W^{\prime}$ boson, with a mass lying in the $1-2 \mathrm{TeV}$ range, not excluded by the results of the LHC.

\section{Scotino DM signal at colliders}

In this section we explore the implications at the LHC of the cosmology-favoured scenarios that have emerged from our dark matter analysis. We choose three benchmark scenarios consistent with the constraints previously studied and provide their definition in terms of the model free parameters in the upper panel of table 3. As detailed in section 3, the scalar 
potential parameter $\lambda_{2}=0$ for all scenarios. Moreover, the small $\lambda_{3}$ value, together with the equality of all $\alpha_{i}$ parameters and the moderate $\kappa$ value, implies that the $A_{1}^{0}, H_{1}^{0}$ and $H_{2}^{ \pm}$Higgs bosons are quite light (as derived from the relations presented in appendix A). We have also chosen scenarios with a small $g_{R}$ value close to the theoretically allowed limit, which guarantees a light $W^{\prime}$-boson (see section 4 ) and induces $v^{\prime} \approx v_{R} \sim 7-8 \mathrm{TeV}$. The breaking of the $\mathrm{SU}(2)_{R^{\prime}} \times \mathrm{U}(1)_{B-L}$ symmetry at such a scale naturally leads to a $Z^{\prime}$-boson mass of about $5 \mathrm{TeV}$ for all benchmark scenarios and a $W^{\prime}$-boson mass of about $1.5 \mathrm{TeV}$. This is more precisely shown in the lower and middle panels of table 3 in which we present the masses of all new physics fields. In the selection of our benchmark points, we impose the lightest scotino to have a mass in the [700-1050] GeV mass window, the BM I scenario focusing on a lighter DM option $\left(m_{n_{\mathrm{DM}}} \approx 750 \mathrm{GeV}\right)$ and the two other scenarios on a heavier setup $\left(m_{n_{\mathrm{DM}}} \approx 900 \mathrm{GeV}\right)$. As discussed in section 5 , many Higgs states are quite light, with masses of about $200 \mathrm{GeV}$ (BM I and BM III scenarios) or $100 \mathrm{GeV}$ (BM II scenario). In addition, our benchmark points choice is LHC-driven, so that we target spectra in which the exotic down-type quarks are heavier than the $W^{\prime}$-boson so that a typical model signature could consist of $W^{\prime}$-boson pairs produced in association with jets through the $p p \rightarrow d^{\prime} d^{\prime} \rightarrow W^{\prime} j W^{\prime} j$ process, for instance.

An interesting feature of the model concerns the lightest charged Higgs boson $H_{2}^{ \pm}$, that, from the LHC perspective, is long-lived, so that previous studies [31] are inapplicable. As seen in table 4, the $H_{2}^{ \pm}$decay width is indeed of about $2 \times 10^{-18} \mathrm{GeV}$ for the $\mathbf{B M} \mathbf{I}$ and BM III scenarios, and of $2 \times 10^{-20} \mathrm{GeV}$ for the $\mathbf{B M}$ II case, so that those scenarios could be probed by searches for heavy stable charged particles (HSCP), the $H_{2}^{ \pm}$bosons being pair-produced via the Drell-Yan mechanism. The corresponding cross sections are given in table 4, for proton-proton collisions at centre-of-mass energies of 7, 8 and $13 \mathrm{TeV}$ and for electron-positron collisions at a centre-of-mass energy of $183 \mathrm{GeV}$. As the $H_{2}^{ \pm}$boson is lighter in the BM II scenario than in the other two scenarios, the associated predictions are larger in the BM II case. For instance, for proton-proton collisions at $13 \mathrm{TeV}$, the total production rate hence reaches about $414 \mathrm{fb}$, compared to about $18 \mathrm{fb}$ for the BM I and BM III cases.

The related searches in $13 \mathrm{TeV}$ LHC collisions exclude signal cross sections ranging from 10 to $100 \mathrm{fb}$, the exact limit value depending on the model [67-72]. The cross sections associated with BM I and BM III $H_{2}^{ \pm}$-boson pair production lie at the border of the stau exclusion limits, so that it is possible that two those benchmark scenarios are excluded. However, a direct transposition of the limits is not straightforward as a consequence of the modeling of various detector effects, which renders any conclusive statement complicated. Similar conclusions hold for 7 and $8 \mathrm{TeV}$ LHC search results [73-75]. On the other hand, all those searches specifically target HSCP with masses larger than $100 \mathrm{GeV}$, so that they are unsensitive to the BM II scenario. For the latter, one must thus rely on LEP results, covering the $[45.9,89.5] \mathrm{GeV}$ mass range [76]. Upper limits on typical HSCP signal cross sections of $0.05-0.19 \mathrm{pb}$ have been extracted from data, but again for models different from the one investigated in this work. Such a model dependence in the results once again prevents us from reinterpreting the results in the ALRSM framework. As HSCP search results may consist in a very general smoking gun on the model, we strongly encourage 
the LHC experimental collaborations to provide information allowing one to recast of their search precisely enough, as to be able to provide limits for the model considered in this work. In the meantime, we focus on other probes for the model.

The heavier charged Higgs state $H_{1}^{ \pm}$could in principle be constrained by more standard searches for additional Higgs states, such as the one of ref. [77]. Those searches are however always targeting a specific production mode and a given decay channel which are not relevant in the cosmology-favoured ALRSM case. For example, the CMS [77] and ATLAS [78] collaborations have investigated the LHC sensitivity to a charged Higgs boson decaying in the $H^{ \pm} \rightarrow \tau^{ \pm} \nu_{\tau}$ mode. In the heavy $H_{1}^{ \pm}$case (scenarios BM I and BM III), cross sections of a few fbs are excluded whilst in the light case (BM II scenario), the analysis targets charged Higgs boson production from the rare decay of a top quark. For heavier charged Higgs bosons, analyses of charged Higgs boson production and decay in a $t b$ final state or heavy Higgs boson production in association with a $t b$ pair or a $W b b$ system have also been carried on (see, e.g., refs. [79, 80]).

We have compared, for all the experimentally relevant signatures, the corresponding predictions (reported in table 4) in the considered ALRSM scenarios with the most recent bounds. The cross sections excluded at the $95 \%$ confidence level have been found to be orders of magnitude larger than our model predictions. Similarly, we have verified that the corresponding mass ranges (for the heavy stable $H_{2}$ state) are not excluded by LEP [81].

The light neutral states $H_{1}^{0}$ and $A_{1}^{0}$ are also long-lived, and can therefore leads to a missing-energy signatures (as they cannot decay into lepton or quark pairs). However, in the corresponding considered spectrum, they can only be produced from rare decays of exotic quarks, so that this gives rise to signatures potentially worth investigating in order to discover or exclude the model. In the following, we focus instead on more abundantly produced final states.

In table 5, we present, for each of the considered benchmark scenarios, predictions for the dark matter features studied in section 5. Each scenario leads to predictions compatible with the cosmological experimental bounds by virtue of a different dynamics. In the first BM I scenario, the DM annihilation cross section is dominated by annihilations into Higgsboson pairs $(\sim 60 \%)$ as well as into pairs of SM gauge bosons $(\sim 35 \%)$, and fermions to a smaller extent. Such an annihilation pattern is typical of light scotino DM setups, as illustrated in the figure 2. In the BM II scenario, DM annihilates essentially in $W^{\prime \mp} H_{2}^{ \pm}$ systems, whilst in the BM III scenario, it dominantly annihilates into pairs of SM charged leptons $(\sim 50 \%)$, quarks $(\sim 30 \%)$ and neutrinos $(\sim 15 \%)$. The BM II and BM III scenarios hence illustrate the two classes of viable scenarios emerging from more moderately heavy scotino dark matter $\left(m_{n_{\mathrm{DM}}} \in[800,1000] \mathrm{GeV}\right)$.

In table 6 , we show predictions relevant for the LHC phenomenology at a centre-ofmass energy of $13 \mathrm{TeV}$ for our three benchmark scenarios. Production cross sections for various processes involving new physics states are presented in the upper panel, whilst the middle and lower panels include the dominant branching ratios of the extra gauge bosons and exotic down-type quarks. We ignore monojet production via the associated production of a scotino pair with a hard jet as this process occurs at a too small rate $(\mathcal{O}(1)$ fb for an optimistic $100 \mathrm{GeV}$ requirement on the leading jet). Other new physics 


\begin{tabular}{|l|c|c|c|}
\hline Benchmarks & BM I & BM II & BM III \\
\hline$\Gamma\left(H_{1}^{ \pm}\right)[\mathrm{GeV}]$ & 3.07 & $1.9 \times 10^{-3}$ & 3.07 \\
$\sigma\left(p p \rightarrow H_{1}^{ \pm}\right) @ 13 \mathrm{TeV}[\mathrm{pb}]$ & $6.503 \times 10^{-5}$ & 0.04352 & $6.901 \times 10^{-5}$ \\
$\sigma\left(p p \rightarrow H_{1}^{ \pm} W^{\mp} b \bar{b}\right) @ 13 \mathrm{TeV}[\mathrm{pb}]$ & $2.723 \times 10^{-3}$ & 2.44 & $2.919 \times 10^{-3}$ \\
$\sigma\left(p p \rightarrow H_{1}^{ \pm} t \bar{b}+\right.$ h.c. $) @ 13 \mathrm{TeV}[\mathrm{pb}]$ & $2.664 \times 10^{-3}$ & 2.374 & $2.859 \times 10^{-3}$ \\
\hline$\Gamma\left(H_{2}^{ \pm}\right)[\mathrm{GeV}]$ & $1.93 \times 10^{-18}$ & $2.62 \times 10^{-20}$ & $1.85 \times 10^{-18}$ \\
$\sigma\left(p p \rightarrow H_{2}^{ \pm} H_{2}^{\mp}\right) @ 7 \mathrm{TeV}[\mathrm{fb}]$ & 5.412 & 163.3 & 5.588 \\
$\sigma\left(p p \rightarrow H_{2}^{ \pm} H_{2}^{\mp}\right) @ 8 \mathrm{TeV}[\mathrm{fb}]$ & 7.153 & 199.8 & 7.392 \\
$\sigma\left(p p \rightarrow H_{2}^{ \pm} H_{2}^{\mp}\right) @ 13 \mathrm{TeV}[\mathrm{fb}]$ & 18.18 & 414.7 & 18.71 \\
$\sigma\left(e e \rightarrow H_{2}^{ \pm} H_{2}^{\mp}\right) @ 183 \mathrm{GeV}[\mathrm{fb}]$ & - & 161.1 & - \\
\hline$B R\left(H_{1}^{ \pm} \rightarrow t \bar{b}\right)$ & $99.6 \%$ & - & $99.6 \%$ \\
$B R\left(H_{1}^{ \pm} \rightarrow W b \bar{b}\right)$ & - & $80.5 \%$ & - \\
$B R\left(H_{1}^{ \pm} \rightarrow c \bar{s}\right)$ & - & $8.9 \%$ & - \\
$B R\left(H_{1}^{ \pm} \rightarrow \tau \nu\right)$ & - & $4.83 \%$ & - \\
$B R\left(H_{1}^{ \pm} \rightarrow c \bar{b}\right)$ & - & $2.1 \%$ & - \\
\hline
\end{tabular}

Table 4. Properties of the light charged Higgs states for the BM I, BM II and BM III benchmark scenarios.

\begin{tabular}{|c|cccc|}
\hline & $\Omega_{\mathrm{DM}} h^{2}$ & $\sigma_{\mathrm{SI}}^{\text {proton }}[\mathrm{pb}]$ & $\sigma_{\mathrm{SI}}^{\text {neutron }}[\mathrm{pb}]$ & $\langle\sigma v\rangle\left[\mathrm{cm}^{3} \mathrm{~s}^{-1}\right]$ \\
\hline BM I & 0.118 & $8.08 \times 10^{-10}$ & $2.88 \times 10^{-11}$ & $7.81 \times 10^{-28}$ \\
BM II & 0.120 & $8.09 \times 10^{-10}$ & $8.37 \times 10^{-10}$ & $3.29 \times 10^{-27}$ \\
BM III & 0.119 & $7.72 \times 10^{-10}$ & $3.67 \times 10^{-11}$ & $1.17 \times 10^{-27}$ \\
\hline
\end{tabular}

Table 5. Predictions, for the BM I, BM II and BM III scenarios, of the observables discussed in our dark matter analysis of the previous section.

processes generally occur at a larger rate, as shown in the table. For all three scenarios, $Z^{\prime}$-boson production is small enough relatively to the LHC limits (by construction of our benchmarks). The rate is hence of about $0.15 \mathrm{fb}$ after accounting for the $Z^{\prime}$-boson branching ratio into electron and muon pairs, $\operatorname{BR}\left(Z^{\prime} \rightarrow \ell \ell\right) \sim 17 \%$ for $\ell$ equivalently denoting an electron or a muon. Consequently this makes the $Z^{\prime}$ signal difficult to observe, even with more luminosity. As the $W^{\prime}$-boson only couples to SM up-type quarks and exotic downtype quarks, it cannot be singly produced. We therefore focus on other processes typical of the ALRSM that instead involve pairs of $W^{\prime}$ bosons and exotic $d^{\prime}$ quarks. The production of a pair of $W^{\prime}$-bosons leads to the production of multileptonic systems in association with missing transverse energy carried away by scotinos, as illustrated by the branching ratio information of the middle panel of table 6 . The total $W^{\prime}$-boson branching ratio into leptons and scotinos $\mathrm{BR}\left(W^{\prime} \rightarrow \ell n_{\mathrm{DM}}\right)$ reaches $20-30 \%$ in all three scenarios, after including the subdominant tau-lepton contribution. The resulting signal cross section (including the branching ratio into a lepton-scotino pair) is then about $0.010 \mathrm{fb}$. Such a rate is far beyond 


\begin{tabular}{|c|cccc|}
\hline & $\sigma\left(p p \rightarrow Z^{\prime}\right)[\mathrm{fb}]$ & $\sigma\left(p p \rightarrow W^{\prime} W^{\prime}\right)[\mathrm{fb}]$ & $\sigma\left(p p \rightarrow W^{\prime} d^{\prime}\right)[\mathrm{fb}]$ & $\sigma\left(p p \rightarrow d^{\prime} d^{\prime}\right)[\mathrm{fb}]$ \\
\hline BM I & 0.821 & 0.0458 & 0.574 & 1.65 \\
BM II & 0.871 & 0.0672 & 1.080 & 2.72 \\
BM III & 0.810 & 0.0465 & 0.564 & 1.61 \\
\hline
\end{tabular}

\begin{tabular}{|c|c|ccc|}
\hline & $\mathrm{BR}\left(Z^{\prime} \rightarrow \ell \ell\right)$ & $\mathrm{BR}\left(W^{\prime} \rightarrow e n_{\mathrm{DM}}\right)$ & $\mathrm{BR}\left(W^{\prime} \rightarrow \mu n_{\mathrm{DM}}\right)$ & $\mathrm{BR}\left(W^{\prime} \rightarrow \tau n_{\mathrm{DM}}\right)$ \\
\hline BM I & 0.166 & 0.203 & 0.054 & 0.020 \\
BM II & 0.167 & 0.158 & 0.056 & 0.016 \\
BM III & 0.171 & 0.178 & 0.063 & 0.018 \\
\hline
\end{tabular}

\begin{tabular}{|c|cccc|}
\hline & $\mathrm{BR}\left(d^{\prime} \rightarrow W^{\prime} u\right)$ & $\mathrm{BR}\left(d^{\prime} \rightarrow W^{\prime} c\right)$ & $\mathrm{BR}\left(d^{\prime} \rightarrow H_{2}^{ \pm} u\right)$ & $\mathrm{BR}\left(d^{\prime} \rightarrow H_{2}^{ \pm} t\right)$ \\
\hline BM I & 0.764 & 0.041 & 0.089 & 0.047 \\
BM II & 0.919 & 0.049 & 0.014 & $\approx 0$ \\
BM III & 0.764 & 0.041 & 0.089 & 0.048 \\
\hline
\end{tabular}

Table 6. Predictions, for the BM I, BM II and BM III scenarios, of various quantities relevant for the associated LHC phenomenology at a centre-of-mass energy of $13 \mathrm{TeV}$. In our notation, $\ell$ equivalently denotes an electron or a muon.

the reach of typical multileptons plus missing energy searches at the LHC, as confirmed by reinterpreting $[82,83]$ and extrapolating [84] the results of the CMS search of ref. [85] targeting electroweak superpartner production and decay in the leptons plus missing energy mode to $3 \mathrm{ab}^{-1}$ with MADANALYsis $5 .^{3}$ This signal, featuring a production times decay rate observable in the 10 ab range at the LHC (for a centre-of-mass energy of $13 \mathrm{TeV}$ ), could however become visible at future colliders.

The upper panel of table 6 also includes cross sections relevant for $d^{\prime} d^{\prime}$ and $d^{\prime} W^{\prime}$ production. Such processes yield production cross sections in the $1 \mathrm{fb}$ range, which makes them potentially more appealing as a door to observing ALRSM at the LHC. Taking into account the large $d^{\prime} \rightarrow W^{\prime} j$ branching fraction, a key signature of those processes is comprised of two leptons, jets and missing transverse energy carried away by the scotinos emerging from the $W^{\prime}$-boson decays. This signature is also typically expected from supersymmetric squark production and decay, so that the results of supersymmetry searches in the opposite-sign dilepton, jets and missing energy mode could be reinterpreted to constrain the ALRSM. We therefore recast the results of the CMS stop search of ref. [88] with MadAnalysis $5,{ }^{4}$ and extrapolate our findings to $3 \mathrm{ab}^{-1}$. We present our results in figure 5. The LHC significance is evaluated according to two measures, labelled by $s$ and

\footnotetext{
${ }^{3}$ Details on the reimplementation of the CMS electroweak superpartner search of ref. [85] in MADANALYSIS 5 can be found in refs. [86, 87].

${ }^{4}$ Details on the reimplementation of the CMS stop search of ref. [88] in MADANALYsis 5 can be found in refs. [89, 90].
} 
$Z_{A}$, that are given by

$$
\begin{aligned}
s & =\frac{S}{\sqrt{B+\sigma_{B}^{2}}} \\
\text { and } Z_{A} & =\sqrt{2\left[(S+B) \ln \left[\frac{(S+B)\left(S+\sigma_{B}^{2}\right)}{B^{2}+(S+B) \sigma_{B}^{2}}\right]-\frac{B^{2}}{\sigma_{B}^{2}} \ln \left[1+\frac{\sigma_{B}^{2} S}{B\left(B+\sigma_{B}^{2}\right)}\right]\right]},
\end{aligned}
$$

where the number of selected signal and background events are denoted by $S$ and $B \pm \sigma_{\mathrm{B}}$ respectively. The first method $(s)$ is rather standard, whereas the second one $\left(Z_{A}\right)$ is more adapted to small numbers of background events [91]. Moreover we consider a signal where both the $W^{\prime} d^{\prime}$ and the $d^{\prime} d^{\prime}$ channels contribute. It turns out that while the LHC has currently very little sensitivity to the signal (i.e. with $36 \mathrm{fb}^{-1}$ ), sensitivity levels of about $3 \sigma$ (for the BM I and BM III scenarios) to $5 \sigma$ (BM II scenario) could be reached at its high-luminosity operation phase (i.e. with $3000 \mathrm{fb}^{-1}$ ) with a conservative level of systematical uncertainties of $20 \%$. In the figure, we also show how a better understanding of the background (corresponding to reduced uncertainties) could guarantee a discovery with luminosities as low as about $750 \mathrm{fb}^{-1}$ ( $5 \%$ of systematics) or $1500 \mathrm{fb}^{-1}$ ( $10 \%$ of systematics) for the most optimistic BM II scenario. For the two other more difficult to observe scenarios, the signal is suppressed so that luminosities of about $1500-2000 \mathrm{fb}^{-1}$ should be necessary for a discovery with a level of $5 \%$ systematics.

\section{Summary and conclusions}

The Standard Model is plagued by several theoretical inconsistencies, while being confirmed by experiments to a high degree of accuracy. Still, there are at least two outstanding experimental facts which the SM does not explain: neutrino masses and dark matter. The standard left-right symmetric model (LRSM) naturally incorporates neutrino masses. However, without ad hoc additional particles it does not include any viable dark matter candidate. We have considered in this work an alternative realisation of the left-right symmetric model, the so-called ALRSM, that can also be obtained from the breaking of an $E_{6}$ Grand Unified setup. Such a class of models has the advantage to offer naturally solutions for both neutrino masses and dark matter problems of the SM. Unlike in the LRSM, in ALRSM the $\mathrm{SU}(2)_{R^{\prime}}$ doublets of right-handed fermions contain exotic states, namely down-type-like quarks $d^{\prime}$ in the quark sector, and neutrino-like scotinos $n$ in the lepton sector. The latter, being part of a doublet, couples to the extra $W^{\prime}$ and $Z^{\prime}$ bosons. In this work, we have shown that this property of the scotino is sufficient to promote it as a bona fide dark matter candidate. Its gauge couplings indeed allow for a sufficient increase in the DM annihilation cross section so that the relic density, as measured by the Planck collaboration, can be accommodated.

Imposing various constraints on the model, such as requiring a cosmology compatible with data (relic density, DM direct and indirect detection) and extra gauge bosons not excluded by the LHC results, we have shown that scotino DM must have a mass in a relatively narrow range of $750-1000 \mathrm{GeV}$ (while ignoring heavier options less appealing 

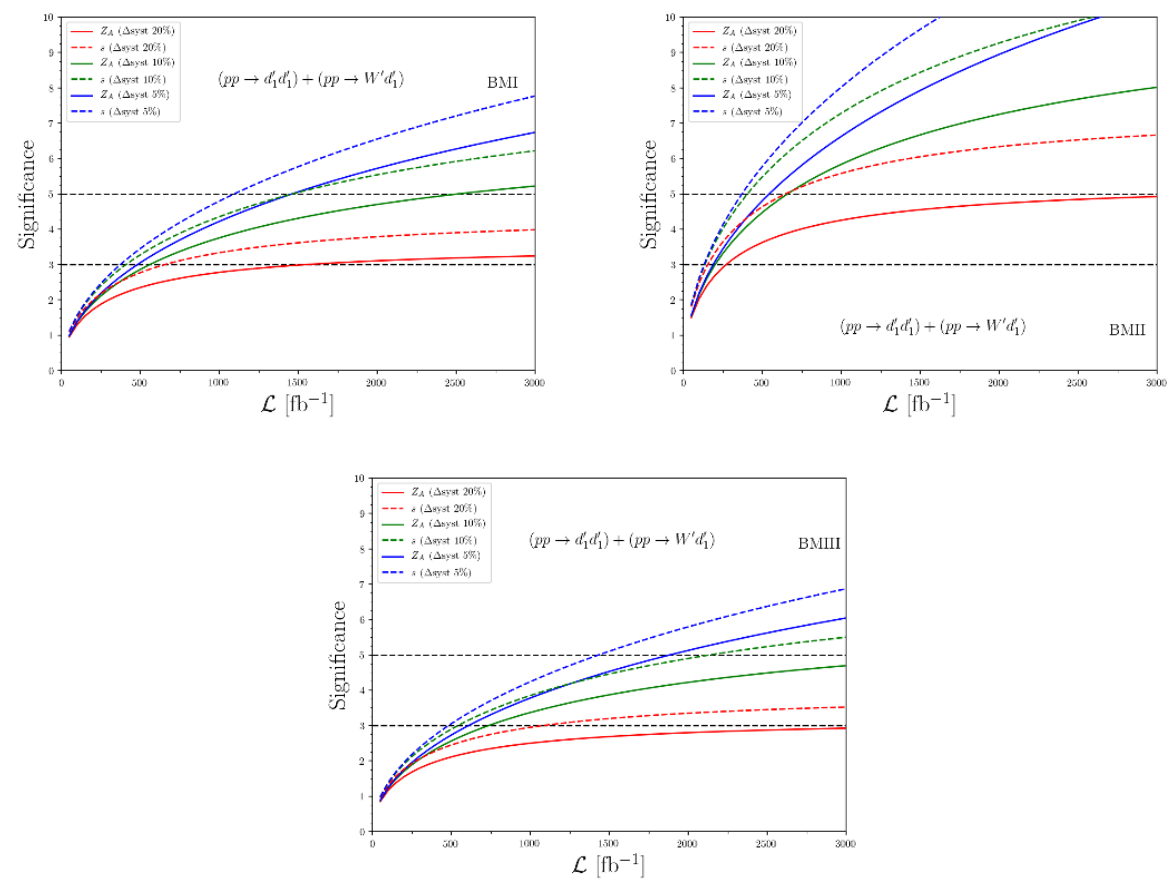

Figure 5. LHC sensitivity to a signature comprised of a dilepton, jets and missing energy in the context of the BM I (upper left), BM II (upper right) and BM III (lower) scenarios. We present our results as a function of the luminosity and recast the CMS stop search of ref. [88], and plot the two significance measures of eq. (6.1).

from the point of view of new physics at current collider experiments). In addition, this restriction imposes strict mass bounds on several of the Higgs bosons of the model. In particular, at least one scalar, one pseudoscalar and one charged Higgs boson have to be light, in the $100-400 \mathrm{GeV}$ mass regime. Moreover, the $W^{\prime}$ gauge boson does not couple to pairs of ordinary fermions so that its mass is mostly unconstrained, unlike the one of the $W_{R}$ boson of the usual LRSM. The only existing bounds arise indirectly, from limits on the $Z^{\prime}$-boson mass derived from its non-observation in LHC data. This however still allows the $W^{\prime}$ boson to be light, with a mass of $\mathcal{O}(1) \mathrm{TeV}$. The model also predicts additional light Higgs states. Given the structure of the model, they however evade all present collider bounds. Of these, a light charged Higgs boson is expected to be long lived, while neutral states would manifest themselves as missing transverse energy at colliders.

We have devised three benchmark scenarios and studied the possibility of observing those DM-favoured ALRSM realisations at the LHC. We have tested the relevance of the ALRSM signatures arising from the $p p \rightarrow W^{\prime} W^{\prime}, W^{\prime} d^{\prime}$ and $d^{\prime} d^{\prime}$ processes. For our choice of spectra, we have shown that the latter two processes have similar cross sections, so that they could both provide an opportunity for the discovery of the ALRSM at the LHC. Out of the three benchmarks, the most promising one can indeed yield a $5 \sigma$ discovery within the future high-luminosity run of the HL-LHC, the exactly luminosity needed depending on assumptions made on the systematic errors. The two other scenarios, associated with 
smaller cross sections, are harder to probe but good prospects are foreseen provided one gets a better control of the background. On the other hand, HSCP searches could possibly consist in smoking guns on the model, provided that future results are either directly interpreted in the ALRSM framework or are released together with enough information for a proper recasting.

In summary, the ALRSM analysed here has numerous attractive features once we impose that its cosmological properties accommodate data: light Higgs bosons, a light charged gauge boson, neutrino masses, and a viable dark matter candidate. The latter in particular renders the spectrum well-defined. In addition, such ALRSM scenarios emerge naturally from a grand unified $E_{6}$ theory, a promising UV completion of the SM, and they offer the promise of being detectable at the high-luminosity LHC.

\section{Acknowledgments}

The authors are grateful to S. Chakraborti and P. Poulose and for enlightening discussions in the early stages of this work, and to J. Araz for useful comments and suggestions. Parts of our numerical calculations have been performed using the High Performance Computing (HPC) server managed by Calcul Québec and Compute Canada. The work of MF and ÖÖ has been partly supported by NSERC through the grant number SAP105354, and the work of BF has been partly supported by French state funds managed by the Agency Nationale de la Recherche (ANR), in the context of the LABEX ILP (ANR-11-INDEX0004-02, ANR-10-LAB-63). ÖÖ thanks the University of Southampton, where part of this work was completed, for their hospitality.

\section{A Diagonalisation of the scalar sector}

The scalar potential $V_{\mathrm{H}}$ of eq. (2.4) is bounded from below if

$$
\lambda_{1} \geq 0, \quad \lambda_{2} \leq 0, \quad \lambda_{3} \geq 0, \quad \alpha_{12} \geq 0, \quad \alpha_{13} \geq 0 \quad \text { and } \quad \alpha_{2}-\alpha_{3} \geq 0,
$$

where $\alpha_{i j}=\alpha_{i}+\alpha_{j}$, and if one of the following conditions is realised,

$$
\left[\lambda_{12} \geq 0\right] \quad \text { or } \quad\left[\lambda_{12} \leq 0, \quad \lambda_{1}+\lambda_{2} \geq 0 \quad \text { and } \quad \lambda_{1}^{2}+4 \lambda_{2}^{2}+8 \lambda_{1} \lambda_{2} \leq 0\right],
$$

with $\lambda_{12}=\lambda_{1}+2 \lambda_{2}$. Moreover, its minimisation allows for the reduction of the number of degrees of freedom of the Higgs sector by three,

$$
\mu_{1}^{2}=\alpha_{12}\left(v_{L}^{2}+v_{R}^{2}\right)+k^{2} \lambda_{1}+\frac{\kappa v_{L} v_{R}}{\sqrt{2} k}, \quad \mu_{2}^{2}=\alpha_{12} k^{2}+\lambda_{3}\left(v_{L}^{2}+v_{R}^{2}\right), \quad \lambda_{4}=\lambda_{3}-\frac{\kappa k}{\sqrt{2} v_{L} v_{R}} .
$$

Focusing first on the charged scalar sector, the squared mass matrix turns out to be block diagonal. The $\phi_{1}^{ \pm}$and $\chi_{L}^{ \pm}$fields therefore mix independently from the $\phi_{2}^{ \pm}$and $\chi_{R}^{ \pm}$fields, as shown by eq. (2.8). The corresponding $2 \times 2$ blocks of the mass matrix $\left(\mathcal{M}_{L}^{ \pm}\right)^{2}$ and $\left(\mathcal{M}_{R}^{ \pm}\right)^{2}$ are written, respectively, in the $\left(\phi_{2}^{ \pm}, \chi_{L}^{ \pm}\right)$and $\left(\phi_{1}^{ \pm}, \chi_{R}^{ \pm}\right)$bases, as

$$
\left(\mathcal{M}_{L, R}^{ \pm}\right)^{2}=\left(\begin{array}{rr}
-\left(\alpha_{2}-\alpha_{3}\right) v_{L, R}^{2}-\frac{\kappa v_{L} v_{R}}{\sqrt{2} k} & \left(\alpha_{2}-\alpha_{3}\right) k v_{L, R}+\frac{\kappa v_{R, L}}{\sqrt{2}} \\
\left(\alpha_{2}-\alpha_{3}\right) k+\frac{\kappa v_{R, L}}{\sqrt{2}} & -\left(\alpha_{2}-\alpha_{3}\right) k^{2}-\frac{\kappa k v_{R, L}}{\sqrt{2} v_{L, R}}
\end{array}\right),
$$


and are diagonalised by the rotations of eq. (2.8). The corresponding mass eigenvalues $M_{H_{1}^{ \pm}}$and $M_{H_{2}^{ \pm}}$are

$$
\begin{aligned}
M_{H_{1}^{ \pm}} & =\frac{k^{2}+v_{L}^{2}}{2 k v_{L}}\left[-2\left(\alpha_{2}-\alpha_{3}\right) k v_{L}-\sqrt{2} \kappa v_{R}\right] \\
\text { and } \quad M_{H_{2}^{ \pm}} & =\frac{k^{2}+v_{R}^{2}}{2 k v_{R}}\left[-2\left(\alpha_{2}-\alpha_{3}\right) k v_{R}-\sqrt{2} \kappa v_{L}\right] .
\end{aligned}
$$

As $\alpha_{2}-\alpha_{3} \geq 0$ from eq. (A.1), forbidding tachyonic fields yields $\kappa<0$. This further implies $\lambda_{4} \geq 0$ by virtue of eq. (A.3). As shown by eq. (2.7), the pseudoscalar and scalar components of the $\phi_{1}^{0}$ field do not mix and consist of the physical $H_{1}^{0}$ and $A_{1}^{0}$ eigenstates. They are mass-degenerate, with masses $M_{H_{1}^{0}}$ and $M_{A_{1}^{0}}$ reading

$$
M_{H_{1}^{0}}^{2}=M_{A_{1}^{0}}^{2}=-\left(\alpha_{2}-\alpha_{3}\right)\left(v_{L}^{2}+v_{R}^{2}\right)-\frac{\kappa v_{L} v_{R}}{\sqrt{2} k}+2 k^{2} \lambda_{2}
$$

The squared mass matrices $\left(\mathcal{M}_{\Re}^{0}\right)^{2}$ and $\left(\mathcal{M}_{\Im}^{0}\right)^{2}$ of the three remaining scalar and pseudoscalar fields are respectively given, in the $\left(\Re\left\{\phi_{2}^{0}\right\}, \Re\left\{\chi_{L}^{0}\right\}, \Re\left\{\chi_{R}^{0}\right\}\right)$ and $\left(\Im\left\{\phi_{2}^{0}\right\}, \Im\left\{\chi_{L}^{0}\right\}, \Im\left\{\chi_{R}^{0}\right\}\right)$ bases, by

$$
\begin{aligned}
\left(\mathcal{M}_{\Re}^{0}\right)^{2} & =\left(\begin{array}{ccc}
2 k^{2} \lambda_{1}-\frac{\kappa v_{L} v_{R}}{\sqrt{2} k} & 2 \alpha_{12} k v_{L}+\frac{\kappa v_{R}}{\sqrt{2}} & 2 \alpha_{12} k v_{R}+\frac{\kappa v_{L}}{\sqrt{2}} \\
2 \alpha_{12} k v_{L}+\frac{\kappa v_{R}}{\sqrt{2}} & 2 \lambda_{3} v_{L}^{2}-\frac{\kappa k v_{R}}{\sqrt{2} v_{L}} & 2 \lambda_{3} v_{L} v_{R}-\frac{\kappa k}{\sqrt{2}} \\
2 \alpha_{12} k v_{R}+\frac{\kappa v_{L}}{\sqrt{2}} & 2 \lambda_{3} v_{L} v_{R}-\frac{\kappa k}{\sqrt{2}} & 2 \lambda_{3} v_{R}^{2}-\frac{\kappa v_{L}}{\sqrt{2} v_{R}}
\end{array}\right), \\
\left(\mathcal{M}_{\Im}^{0}\right)^{2} & =\frac{\kappa}{\sqrt{2}}\left(\begin{array}{ccc}
-\frac{v_{L} v_{R}}{k} & v_{R} & -v_{L} \\
v_{R} & -\frac{k v_{R}}{v_{L}} & k \\
-v_{L} & k & -\frac{k v_{L}}{v_{R}}
\end{array}\right),
\end{aligned}
$$

and are diagonalised by the two $U_{3 \times 3}^{\mathrm{H}}$ and $U_{3 \times 3}^{\mathrm{A}}$ rotation matrices of eq. (2.7). These are explicitly given by

$$
\begin{aligned}
U_{3 \times 3}^{\mathrm{A}}=\frac{1}{\sqrt{2}}\left(\begin{array}{ccc}
-\frac{k}{\sqrt{k^{2}+v_{R}^{2}}} & \frac{k v_{R}^{2}}{\sqrt{\left(k^{2}+v_{R}^{2}\right)\left(v_{L}^{2} v_{R}^{2}+k^{2} v_{L}^{2}+v_{R}^{2} k^{2}\right)}} & \frac{v_{R} v_{L}}{\sqrt{v_{L}^{2} v_{R}^{2}+k^{2} v_{L}^{2}+v_{R}^{2} k^{2}}} \\
0 & v_{L} \frac{\sqrt{k^{2}+v_{R}^{2}}}{\sqrt{v_{L}^{2} v_{R}^{2}+k^{2} v_{L}^{2}+v_{R}^{2} k^{2}}} & -\frac{k v_{R}}{\sqrt{v_{L}^{2} v_{R}^{2}+k^{2} v_{L}^{2}+v_{R}^{2} k^{2}}} \\
\frac{v_{R}}{\sqrt{v_{R}^{2}+k^{2}}} & \frac{k^{2} v_{R}}{\sqrt{\left(k^{2}+v_{R}^{2}\right)\left(v_{L}^{2} v_{R}^{2}+k^{2} v_{L}^{2}+v_{R}^{2} k^{2}\right)}} & \frac{k v_{L}}{\sqrt{v_{L}^{2} v_{R}^{2}+k^{2} v_{L}^{2}+v_{R}^{2} k^{2}}}
\end{array}\right), \\
U_{3 \times 3}^{\mathrm{H}}=\frac{1}{\sqrt{2}}\left(\begin{array}{lll}
\frac{f_{0}}{\sqrt{\mathcal{D}_{1}}} \frac{f_{2}\left(1+g_{0}^{2}\right)-f_{0}\left(1+g_{0} g_{2}\right)}{\sqrt{\mathcal{D}_{1} \mathcal{D}_{2}}} & \frac{\xi\left(g_{2}-g_{0}\right)}{\sqrt{\mathcal{D}_{2}}} \\
\frac{g_{0}}{\sqrt{\mathcal{D}_{1}}} & \frac{g_{2}\left(1+f_{0}^{2}\right)-g_{0}\left(1+f_{0} f_{2}\right)}{\sqrt{\mathcal{D}_{1} \mathcal{D}_{2}}} & \frac{\xi\left(f_{0}-f_{2}\right)}{\sqrt{\mathcal{D}_{2}}} \\
\frac{1}{\sqrt{\mathcal{D}_{1}}} & \frac{f_{0}^{2}+g_{0}^{2}-f_{0} f_{2}-g_{0} g_{2}}{\sqrt{\mathcal{D}_{1} \mathcal{D}_{2}}} & \frac{\xi\left(g_{0} f_{2}-g_{2} f_{0}\right)}{\sqrt{\mathcal{D}_{2}}}
\end{array}\right)
\end{aligned}
$$


and depend on various functions of the Higgs mass eigenvalues $M_{H_{i}^{0}}$,

$$
\begin{aligned}
f_{i} & =\frac{2 M_{H_{i}^{0}}^{4} v_{L} v_{R}+M_{H_{i}^{0}}^{2}\left(v_{L}^{2}+v_{R}^{2}\right)\left(\sqrt{2} k \kappa-4 v_{L} v_{R} \lambda_{3}\right)-2 \sqrt{2} k\left(v_{L}^{2}-v_{R}^{2}\right)^{2} \lambda_{3} \kappa}{v_{R}\left[M_{H_{i}^{0}}^{2}\left(4 k v_{L} v_{R} \alpha_{12}+\sqrt{2} v_{L}^{2} \kappa\right)+2 \sqrt{2}\left(k^{2} \alpha_{12}+v_{L}^{2} \lambda_{3}\right)\left(v_{R}^{2}-v_{L}^{2}\right) \kappa\right]}, \\
g_{i} & =\frac{v_{L}}{v_{R}} \frac{M_{H_{i}^{0}}^{2}\left(4 k v_{L} v_{R} \alpha_{12}+\sqrt{2} v_{R}^{2} \kappa\right)+2 \sqrt{2}\left(k^{2} \alpha_{12}+v_{R}^{2} \lambda_{3}\right)\left(v_{L}^{2}-v_{R}^{2}\right) \kappa}{M_{H_{i}^{0}}^{2}\left(4 k v_{L} v_{R} \alpha_{12}+\sqrt{2} v_{L}^{2} \kappa\right)+2 \sqrt{2}\left(k^{2} \alpha_{12}+v_{L}^{2} \lambda_{3}\right)\left(v_{R}^{2}-v_{L}^{2}\right) \kappa}, \\
\mathcal{D}_{1} & =1+f_{0}^{2}+g_{0}^{2} \\
\mathcal{D}_{2} & =f_{2}^{2}\left(1+g_{0}^{2}\right)+\left(g_{0}-g_{2}\right)^{2}-2 f_{0} f_{2}\left(1+g_{0} g_{2}\right)+f_{0}^{2}\left(1+g_{2}^{2}\right), \\
\xi & =\operatorname{sgn}\left[g_{0}\left(f_{2}-f_{3}\right)+g_{2}\left(f_{3}-f_{0}\right)+g_{3}\left(f_{0}-f_{2}\right)\right] .
\end{aligned}
$$

In our conventions, we trade the $\lambda_{1}$ free parameter of the scalar potential for the mass of the lightest Higgs state $H_{0}^{0}$ (that can then be set freely and thus match the SM Higgs boson mass). $\lambda_{1}$ becomes thus a dependent parameter,

$$
\lambda_{1}=\frac{1}{2 k^{3}} \frac{\sqrt{2} k v_{L} v_{R} M_{H_{0}^{0}}^{6}+\mathfrak{a}^{(4)} M_{H_{0}^{0}}^{4}-2 \mathfrak{a}^{(2)} M_{H_{0}^{0}}^{2}-4 \alpha_{12}^{2} \kappa k^{4}\left(v_{L}^{2}-v_{R}^{2}\right)^{2}}{\sqrt{2} v_{L} v_{R} M_{H_{0}^{0}}^{4}+\left(\kappa k-2 \sqrt{2} \lambda_{3} v_{L} v_{R}\right)\left(v_{L}^{2}+v_{R}^{2}\right) M_{H_{0}^{0}}^{2}-2 \kappa k \lambda_{3}\left(v_{L}^{2}-v_{R}^{2}\right)^{2}},
$$

and the remaining scalar masses then read

$$
\begin{aligned}
M_{A_{2}^{0}}^{2} & =-\frac{\kappa}{\sqrt{2} k v_{L} v_{R}}\left[v_{L}^{2} v_{R}^{2}+k^{2}\left(v_{L}^{2}+v_{R}^{2}\right)\right] \\
\text { and } \quad M_{H_{2,3}^{0}}^{2} & =\frac{1}{2}\left[\mathfrak{a} \pm \sqrt{\mathfrak{a}^{2}+4\left(\mathfrak{b}+\mathfrak{a} M_{H_{0}^{0}}^{2}\right)}\right] .
\end{aligned}
$$

with

$$
\begin{aligned}
& \mathfrak{a}^{(4)}=-2 \sqrt{2} k \lambda_{3} v_{L} v_{R}\left(v_{L}^{2}+v_{R}^{2}\right)+\kappa\left(v_{L}^{2} v_{R}^{2}+k^{2}\left(v_{L}^{2}+v_{R}^{2}\right)\right) \\
& \mathfrak{a}^{(2)}= 2 \sqrt{2} \alpha_{12}^{2} k^{3} v_{L} v_{R}\left(v_{L}^{2}+v_{R}^{2}\right)+\kappa\left(\lambda_{3} v_{L}^{2} v_{R}^{2}\left(v_{L}^{2}+v_{R}^{2}\right)+k^{2}\left[4 \alpha_{12} v_{L}^{2} v_{R}^{2}+\lambda_{3}\left(v_{L}^{2}-v_{R}^{2}\right)^{2}\right]\right), \\
& \mathfrak{a}= \frac{1}{\sqrt{2} k v_{L} v_{R}}\left[v_{L} v_{R}\left(2 \sqrt{2} k^{3} \lambda_{1}-\kappa v_{L} v_{R}\right)+k\left(2 \sqrt{2} \lambda_{3} v_{L} v_{R}-\kappa k\right)\left(v_{L}^{2}+v_{R}^{2}\right)\right]-M_{H_{0}^{0}}^{2} \\
& \mathfrak{b}=\frac{1}{k v_{L} v_{R}}\left[\sqrt{2} \kappa k^{2}\left(4 \alpha_{12} v_{L}^{2} v_{R}^{2}+\lambda_{3}\left(v_{L}^{2}-v_{R}^{2}\right)^{2}\right)\right. \\
&\left.\quad+\left(4 k^{3}\left(\alpha_{12}^{2}-\lambda_{1} \lambda_{3}\right) v_{L} v_{R}+\sqrt{2} \kappa\left(k^{4} \lambda_{1}+\lambda_{3} v_{L}^{2} v_{R}^{2}\right)\right)\left(v_{L}^{2}+v_{R}^{2}\right)\right]
\end{aligned}
$$

\section{B The fermion sector}

Fermion mass terms are generated from the Yukawa Lagrangian of eq. (2.3) after the breaking of the $\mathrm{SU}(2)_{L} \times \mathrm{SU}(2)_{R^{\prime}} \times \mathrm{U}(1)_{B-L}$ symmetry down to electromagnetism,

$$
\begin{aligned}
\mathcal{L}_{\mathrm{F}}^{\text {mass }}= & -\frac{k}{\sqrt{2}}\left[\bar{e}_{L} \hat{\mathbf{Y}}^{e} e_{R}+\bar{u}_{L} \hat{\mathbf{Y}}^{u} u_{R}\right]-\frac{v_{L}}{\sqrt{2}}\left[\bar{d}_{L} \hat{\mathbf{Y}}^{d} d_{R}+\bar{\nu}_{L} \hat{\mathbf{Y}}^{\nu} \nu_{R}\right] \\
& -\frac{v_{R}}{\sqrt{2}}\left[\bar{d}_{R}^{\prime} \hat{\mathbf{Y}}^{d^{\prime}} d_{L}^{\prime}+\bar{n}_{R} \hat{\mathbf{Y}}^{n} n_{L}\right]+\text { h.c. }
\end{aligned}
$$


The different mass matrices $\hat{\mathbf{Y}}$ can be diagonalised through 12 unitary rotations,

$$
\begin{aligned}
& \frac{k}{\sqrt{2}} \hat{\mathbf{Y}}^{u} \rightarrow \frac{k}{\sqrt{2}} V_{u} \mathbf{Y}^{u} U_{u}^{\dagger}=\left(\begin{array}{ccc}
M_{u} & 0 & 0 \\
0 & M_{c} & 0 \\
0 & 0 & M_{t}
\end{array}\right), \\
& \frac{v_{L}}{\sqrt{2}} \hat{\mathbf{Y}}^{d} \rightarrow \frac{v_{L}}{\sqrt{2}} V_{d} \mathbf{Y}^{d} U_{d}^{\dagger}=\left(\begin{array}{ccc}
M_{d} & 0 & 0 \\
0 & M_{s} & 0 \\
0 & 0 & M_{b}
\end{array}\right), \\
& \frac{v_{L}}{\sqrt{2}} \hat{\mathbf{Y}}^{\nu} \rightarrow \frac{v_{L}}{\sqrt{2}} V_{\nu} \mathbf{Y}^{\nu} U_{\nu}^{\dagger}=\left(\begin{array}{ccc}
M_{\nu_{e}} & 0 & 0 \\
0 & M_{\nu_{\mu}} & 0 \\
0 & 0 & M_{\nu_{\tau}}
\end{array}\right) \text {, } \\
& \frac{k}{\sqrt{2}} \hat{\mathbf{Y}}^{e} \rightarrow \frac{k}{\sqrt{2}} V_{e} \mathbf{Y}^{e} U_{e}^{\dagger}=\left(\begin{array}{ccc}
M_{e} & 0 & 0 \\
0 & M_{\mu} & 0 \\
0 & 0 & M_{\tau}
\end{array}\right), \\
& \frac{v_{R}}{\sqrt{2}} \hat{\mathbf{Y}}^{d^{\prime}} \rightarrow \frac{v_{R}}{\sqrt{2}} U_{d^{\prime}} \mathbf{Y}^{d^{\prime}} V_{d^{\prime}}^{\dagger}=\left(\begin{array}{ccc}
M_{d^{\prime}} & 0 & 0 \\
0 & M_{s^{\prime}} & 0 \\
0 & 0 & M_{b^{\prime}}
\end{array}\right) \text {, } \\
& \frac{v_{R}}{\sqrt{2}} \hat{\mathbf{Y}}^{n} \rightarrow \frac{v_{R}}{\sqrt{2}} U_{n} \mathbf{Y}^{n} V_{n}^{\dagger}=\left(\begin{array}{ccc}
M_{n_{e}} & 0 & 0 \\
0 & M_{n_{\mu}} & 0 \\
0 & 0 & M_{n_{\tau}}
\end{array}\right),
\end{aligned}
$$

leading to diagonal and real $\mathbf{Y}$ matrices. These rotations equivalently correspond to replacing the fermion gauge eigenbasis by the physical one,

$$
\begin{array}{rlrlrl}
u_{L} & \rightarrow V_{u} u_{L}, & d_{L} \rightarrow V_{d} d_{L}, & \nu_{L} \rightarrow V_{\nu} \nu_{L}, \\
e_{L} \rightarrow V_{e} e_{L}, & d_{L}^{\prime} \rightarrow V_{d^{\prime}} d_{L}^{\prime}, & n_{L} \rightarrow V_{n} n_{L}, \\
u_{R} \rightarrow U_{u} u_{R}, & d_{R} \rightarrow U_{d} d_{R}, & \nu_{R} \rightarrow U_{\nu} \nu_{R}, \\
e_{R} \rightarrow U_{e} e_{R}, & d_{R}^{\prime} \rightarrow U_{d^{\prime}} d_{R}^{\prime}, & n_{R} \rightarrow U_{n} n_{R} .
\end{array}
$$

As in the SM, conventionally we keep the left-handed up-type quark and charged lepton bases unchanged and absorb the $V_{u}-V_{d}$ and $V_{\nu}-V_{e}$ rotations in a redefinition of the down-type quark and neutrino states. Similarly, the $U_{u}-U_{d^{\prime}}$ and $U_{n}-U_{e}$ rotations are conveniently absorbed in a redefinition of the $d_{R}^{\prime}$ and $n_{L}$ bases, the right-handed up-type quark and charged lepton bases being kept unchanged,

$$
\begin{array}{ll}
d_{L} \rightarrow V_{u}^{\dagger} V_{d} d_{L} \equiv V_{\mathrm{CKM}} d_{L}, & \nu_{L} \rightarrow V_{e}^{\dagger} V_{\nu} \nu_{L} \equiv V_{\mathrm{PMNS}} d_{L}, \\
d_{R}^{\prime} \rightarrow U_{u}^{\dagger} U_{d^{\prime}} d_{R}^{\prime} \equiv V_{\mathrm{CKM}^{\prime}} d_{R}^{\prime}, & n_{R} \rightarrow U_{e}^{\dagger} U_{n} n_{R} \equiv V_{\mathrm{PMNS}^{\prime}} n_{R} .
\end{array}
$$

Omitting any potential Majorana phase, each of the four CKM/PMNS rotation matrices can be defined by three mixing angles $\theta_{i j}$ and a Dirac phase $\delta$.

\section{Technical details on our FeynRules implementation}

We collect the properties of the new physics fields and external parameters associated with our FEYNRULEs implementation of the ALRSM model in tables 7 and 8, where we 


\begin{tabular}{|cccc|}
\hline Field & Spin & Name & PDG \\
\hline$Z^{\prime}$ & 1 & Zp & 32 \\
$W^{\prime+}$ & 1 & $\mathrm{Wp}$ & 34 \\
$n_{i}(i=1,2,3)$ & $1 / 2$ & $\mathrm{nl}$ & $6000012,6000014,6000016$ \\
$d_{i}^{\prime}(i=1,2,3)$ & $1 / 2$ & $\mathrm{dqp}$ & $6000001,6000003,6000005$ \\
$H_{i}^{0}(i=0,1,2,3)$ & 0 & $\mathrm{~h} 0$ & $25,25,45,55$ \\
$A_{i}^{0}(i=1,2)$ & 0 & $\mathrm{~A} 0$ & 36,46 \\
$H_{i}^{+}(i=1,2)$ & 0 & Hp & 37,47 \\
\hline
\end{tabular}

Table 7. Mass eigenstates that supplement the SM, together with their spin quantum number (second column), the name used in the FEYNRULES implementation (third column) and the associated PDG identifier (last column).

\begin{tabular}{|cccc|}
\hline Parameter & Name & LH block & LH counter \\
\hline $\tan \beta$ & tb & SMINPUTS & 5 \\
$g_{R}$ & gR & SMINPUTS & 6 \\
$v^{\prime}$ & vevp & SMINPUTS & 7 \\
\hline$\lambda_{2}$ & lam2 & HPOTINPUTS & 1 \\
$\lambda_{3}$ & lam3 & HPOTINPUTS & 2 \\
$\alpha_{1}$ & alp1 & HPOTINPUTS & 3 \\
$\alpha_{2}$ & alp2 & HPOTINPUTS & 4 \\
$\alpha_{3}$ & alp3 & HPOTINPUTS & 5 \\
$\kappa$ & kap & HPOTINPUTS & 6 \\
\hline$a_{\mathrm{H}}^{g}$ & Ghgg & EFFECTIVEHIGGS & 1 \\
$a_{\mathrm{H}}^{a}$ & Ghaa & EFFECTIVEHIGGS & 2 \\
\hline
\end{tabular}

\begin{tabular}{|cccc|}
\hline Parameter & Name & LH block & LH counter \\
\hline$M_{\nu_{e}}$ & Mve & MASS & 12 \\
$M_{\nu_{\mu}}$ & Mvm & MASS & 14 \\
$M_{\nu_{\tau}}$ & Mvt & MASS & 16 \\
$M_{n_{e}}$ & Mne & MASS & 6000012 \\
$M_{n_{\mu}}$ & Mnm & MASS & 6000014 \\
$M_{n_{\tau}}$ & Mnt & MASS & 6000016 \\
$M_{d^{\prime}}$ & MDP & MASS & 6000001 \\
$M_{s^{\prime}}$ & MSP & MASS & 6000003 \\
$M_{b^{\prime}}$ & MBP & MASS & 6000005 \\
\hline
\end{tabular}

\begin{tabular}{|cccc|}
\hline Parameter & Name & LH block & LH counter \\
\hline$\lambda$ & CKMlam & CKMBLOCK & 1 \\
$A$ & CKMA & CKMBLOCK & 2 \\
$\bar{\rho}$ & CKMrho & CKMBLOCK & 3 \\
$\bar{\eta}$ & CKMeta & CKMBLOCK & 4 \\
\hline$s_{12}^{\left(\mathrm{CKM}^{\prime}\right)}$ & CKMps12 & CKMBLOCK & 11 \\
$s_{23}^{\left(\mathrm{CKM}^{\prime}\right)}$ & CKMps23 CKMBLOCK & 12 \\
$s_{13}^{\left(\mathrm{CKM}^{\prime}\right)}$ & CKMps13 & CKMBLOCK & 13 \\
$\delta_{\mathrm{CKM}^{\prime}}$ & CKMpdel & CKMBLOCK & 14 \\
\hline
\end{tabular}

\begin{tabular}{|cccc|}
\hline Parameter & Name & LH block & LH counter \\
\hline$s_{12}^{\text {(PMNS) }}$ & PMNSs12 & PMNSBLOCK & 1 \\
$s_{23}^{(\text {PMNS })}$ & PMNSs23 & PMNSBLOCK & 2 \\
$s_{13}^{(\text {PMNS })}$ & PMNSs13 & PMNSBLOCK & 3 \\
$\delta_{\text {PMNS }}$ & PMNSdel & PMNSBLOCK & 4 \\
\hline$s_{12}^{\text {(PMNS') }}$ & PMNSps12 PMNSBLOCK & 11 \\
$s_{23}^{\left(\text {PMNS }^{\prime}\right)}$ & PMNSps23 PMNSBLOCK & 12 \\
$s_{13}^{\left(\text {PMNS' }^{\prime}\right)}$ & PMNSps13 PMNSBLOCK & 13 \\
$\delta_{\text {PMNS' }^{\prime}}$ & PMNSpdel PMNSBLOCK & 14 \\
\hline
\end{tabular}

Table 8. New physics external parameters of our ALRSM implementation, together with their name and the Les Houches (LH) block and counter information allowing to change its numerical value on run time. We recall that for consistency, $\kappa<0$ and the conditions of eqs. (A.1) and (A.2) must be satisfied. Those parameters supplement the usual set of electroweak inputs given in the LEP scheme, as well as all SM fermion masses. 
additionally include properties useful for the user when running any programme relying on our implementation.

As can be noticed from the tables, the left-handed and right-handed scotinos are combined to form a Dirac fermion $n_{i}$ (with $i=1,2,3$ being a generation index) and the left-handed and right-handed exotic quarks are combined to form a Dirac fermion $d_{i}^{\prime}$ (with $i=1,2,3$ being again a generation index). Whilst all fermion masses are free parameters of the model (see also appendix B), all boson masses are internal (i.e. are derived parameters), with the exception of the SM Higgs boson mass $M_{H_{0}^{0}}$ (see appendix A) and the $Z$-boson mass. As for the SM implementation included with FeYnRules, our model defines the electroweak sector following the LEP scheme that is known to yield the minimal parametric uncertainty in the predictions. The three electroweak inputs are thus the Fermi coupling $G_{F}$, the fine structure constant $\alpha$ and the $Z$-boson mass $M_{Z}$. The gauge and scalar sectors are then fully defined by fixing nine parameters, that we choose to be $v^{\prime}, t_{\beta}, g_{R}, \lambda_{2}, \lambda_{3}$, $\alpha_{1}, \alpha_{2}, \alpha_{3}$ and $\kappa$. We recall that the user must ensure that the conditions of eqs. (A.1) and (A.2) are satisfied when providing the numerical values of these parameters, and that $\kappa<0$ to avoid tachyonic charged Higgs bosons.

All other parameters of the gauge and Higgs sectors are then derived as follows. The vacuum expectation values $v, v_{L}, v_{R}$ and $k$ are obtained from $G_{F}, v^{\prime}$ and $t_{\beta}$,

$$
v^{2}=\frac{1}{\sqrt{2} G_{F}}, \quad v_{L}=v \cos \beta, \quad k=v \sin \beta \quad \text { and } \quad v_{R}^{2}=v^{\prime 2}-k^{2} .
$$

As in the SM the $W$-boson mass is derived from the electroweak inputs,

$$
M_{W}^{2}=\frac{M_{Z}^{2}}{2}\left[1+\sqrt{1-2 \sqrt{2} \frac{\pi \alpha}{G_{F} M_{Z}^{2}}}\right] \text {, }
$$

so that eq. (2.10) can be used to derive the $\mathrm{SU}(2)_{L}$ gauge coupling $g_{L}$. As $e=\sqrt{4 \pi \alpha}$, one can then derive the hypercharge coupling $g_{Y}$ and the sine and cosine of the electroweak mixing angle $\theta_{W}$ from eq. (2.13), which further allows us to calculate the $B-L$ coupling constant $g_{B-L}$, the cosine of the $\varphi_{W}$ mixing angle and the so far neglected $Z-Z^{\prime}$ mixing. It is up to the user to verify that his/her choice of input parameter yields $\tan \left(2 \vartheta_{W}\right) \lesssim 10^{-3}$. Furthermore, the $W^{\prime}$ - and $Z^{\prime}$-boson masses are obtained from eqs. (2.10) and (2.14), and the other parameters of the Higgs potential (i.e., $\mu_{1}, \mu_{2}, \lambda_{1}$ and $\lambda_{4}$ ) are obtained from eq. (A.3) and eq. (A.10).

In the fermion sector, the various CKM and PNMS matrices are obtained from their standard expressions in terms of three mixing angles and a phase,

$$
V=\left(\begin{array}{ccc}
c_{12} c_{13} & s_{12} c_{13} & s_{13} e^{-i \delta} \\
-s_{12} c_{23}-c_{12} s_{23} s_{13} e^{i \delta} & c_{12} c_{23}-s_{12} s_{23} s_{13} e^{i \delta} & s_{23} c_{13} \\
s_{12} s_{23}-c_{12} c_{23} s_{13} e^{i \delta} & -c_{12} s_{23}-s_{12} c_{23} s_{13} e^{i \delta} & c_{23} c_{13}
\end{array}\right),
$$

where $s_{i j} \equiv \sin \theta_{i j}$ and $c_{i j} \equiv \cos \theta_{i j}$ denote the sine and cosine of the various mixing angles. Concerning the SM CKM matrix, we have however traded the input parameters by the usual Wolfenstein parameters $A, \lambda, \bar{\rho}$ and $\bar{\eta}$,

$$
s_{12}^{(\mathrm{CKM})}=\lambda, \quad s_{23}^{(\mathrm{CKM})}=A \lambda^{2} \quad \text { and } \quad s_{13}^{(\mathrm{CKM})} e^{i \delta_{\mathrm{CKM}}}=\frac{A \lambda^{3} \sqrt{1-A^{2} \lambda^{4}}(\bar{\rho}+i \bar{\eta})}{\sqrt{1-\lambda^{2}}\left[1-A^{2} \lambda^{4}(\bar{\rho}+i \bar{\eta})\right]} .
$$


Open Access. This article is distributed under the terms of the Creative Commons Attribution License (CC-BY 4.0), which permits any use, distribution and reproduction in any medium, provided the original author(s) and source are credited.

\section{References}

[1] G. Arcadi et al., The waning of the WIMP? A review of models, searches and constraints, Eur. Phys. J. C 78 (2018) 203 [arXiv:1703.07364] [inSPIRE].

[2] M. Kawasaki and K. Nakayama, Axions: theory and cosmological role, Ann. Rev. Nucl. Part. Sci. 63 (2013) 69 [arXiv:1301.1123] [INSPIRE].

[3] P.W. Graham et al., Experimental searches for the axion and axion-like particles, Ann. Rev. Nucl. Part. Sci. 65 (2015) 485 [arXiv:1602.00039] [InSPIRE].

[4] Planck collaboration, Planck 2018 results. VI. Cosmological parameters, arXiv: 1807.06209 [INSPIRE].

[5] D. Feldman, B. Körs and P. Nath, Extra-weakly interacting dark matter, Phys. Rev. D 75 (2007) 023503 [hep-ph/0610133] [INSPIRE].

[6] E. Izaguirre, G. Krnjaic, P. Schuster and N. Toro, Analyzing the discovery potential for light dark matter, Phys. Rev. Lett. 115 (2015) 251301 [arXiv:1505.00011] [INSPIRE].

[7] D. Curtin, R. Essig, S. Gori and J. Shelton, Illuminating dark photons with high-energy colliders, JHEP 02 (2015) 157 [arXiv: 1412.0018] [INSPIRE].

[8] R. Essig et al., Working group report: new light weakly coupled particles, in the proceedings of the 2013 Community Summer Study on the Future of U.S. Particle Physics: Snowmass on the Mississippi (CSS2013), July 29-August 6, Minneapolis, U.S.A. (2013), arXiv:1311.0029, http://www.slac.stanford.edu/econf/C1307292/docs/IntensityFrontier/NewLight-17.pdf [INSPIRE].

[9] H. Davoudiasl, H.-S. Lee and W.J. Marciano, 'Dark' Z implications for parity violation, rare meson decays and Higgs physics, Phys. Rev. D 85 (2012) 115019 [arXiv:1203.2947] [INSPIRE].

[10] T. Toma, Internal Bremsstrahlung signature of real scalar dark matter and consistency with thermal relic density, Phys. Rev. Lett. 111 (2013) 091301 [arXiv:1307.6181] [INSPIRE].

[11] F. Giacchino, L. Lopez-Honorez and M.H.G. Tytgat, Scalar dark matter models with significant internal Bremsstrahlung, JCAP 10 (2013) 025 [arXiv: 1307.6480] [INSPIRE].

[12] F. Giacchino, L. Lopez-Honorez and M.H.G. Tytgat, Bremsstrahlung and gamma ray lines in 3 scenarios of dark matter annihilation, JCAP 08 (2014) 046 [arXiv:1405.6921] [INSPIRE].

[13] A. Ibarra, T. Toma, M. Totzauer and S. Wild, Sharp gamma-ray spectral features from scalar dark matter annihilations, Phys. Rev. D 90 (2014) 043526 [arXiv:1405.6917] [INSPIRE].

[14] F. Giacchino et al., Signatures from scalar dark matter with a vector-like quark mediator, JCAP 02 (2016) 002 [arXiv: 1511.04452] [INSPIRE].

[15] S. Colucci et al., Top-philic vector-like portal to scalar dark matter, Phys. Rev. D 98 (2018) 035002 [arXiv: 1804.05068] [INSPIRE].

[16] F. Gursey, P. Ramond and P. Sikivie, A universal gauge theory model based on $E_{6}$, Phys. Lett. B 60 (1976) 177.

[17] Y. Achiman and B. Stech, Quark lepton symmetry and mass scales in an E 6 unified gauge model, Phys. Lett. B 77 (1978) 389. 
[18] J.L. Hewett and T.G. Rizzo, Low-energy phenomenology of superstring inspired $E_{6}$ models, Phys. Rept. 183 (1989) 193 [INSPIRE].

[19] P. Langacker and J. Wang, U(1)' symmetry breaking in supersymmetric $E_{6}$ models, Phys. Rev. D 58 (1998) 115010 [hep-ph/9804428] [INSPIRE].

[20] J.C. Pati and A. Salam, Lepton number as the fourth color, Phys. Rev. D 10 (1974) 275 [Erratum ibid. D 11 (1975) 703] [INSPIRE].

[21] R.N. Mohapatra and J.C. Pati, A natural left-right symmetry, Phys. Rev. D 11 (1975) 2558 [INSPIRE].

[22] G. Senjanović and R.N. Mohapatra, Exact left-right symmetry and spontaneous violation of parity, Phys. Rev. D 12 (1975) 1502 [inSPIRE].

[23] R.N. Mohapatra, F.E. Paige and D.P. Sidhu, Symmetry breaking and naturalness of parity conservation in weak neutral currents in left-right symmetric gauge theories, Phys. Rev. D 17 (1978) 2462 [INSPIRE].

[24] S. Bahrami et al., Dark matter and collider studies in the left-right symmetric model with vectorlike leptons, Phys. Rev. D 95 (2017) 095024 [arXiv:1612.06334] [INSPIRE].

[25] E. Ma, Particle dichotomy and left-right decomposition of $E_{6}$ superstring models, Phys. Rev. D 36 (1987) 274 [INSPIRE].

[26] M. Frank, I. Turan and M. Sher, Neutrino masses in the effective rank-5 subgroups of $E_{6}$ : supersymmetric case, Phys. Rev. D 71 (2005) 113002 [hep-ph/0503084] [INSPIRE].

[27] K.S. Babu, X.-G. He and E. Ma, New supersymmetric left-right gauge model: Higgs boson structure and neutral current analysis, Phys. Rev. D 36 (1987) 878 [INSPIRE].

[28] E. Ma, Dark left-right model: CDMS, LHC, ETC, J. Phys. Conf. Ser. 315 (2011) 012006 [arXiv: 1006.3804] [INSPIRE].

[29] G. Barenboim, J. Bernabeu, J. Prades and M. Raidal, Constraints on the $W_{R}$ mass and CP-violation in left-right models, Phys. Rev. D 55 (1997) 4213 [hep-ph/9611347] [INSPIRE].

[30] S. Khalil, H.-S. Lee and E. Ma, Bound on $Z^{\prime}$ mass from CDMS II in the dark left-right gauge model II, Phys. Rev. D 81 (2010) 051702 [arXiv: 1002.0692] [INSPIRE].

[31] M. Ashry and S. Khalil, Phenomenological aspects of a TeV-scale alternative left-right model, Phys. Rev. D 91 (2015) 015009 [arXiv:1310.3315] [InSPIRE].

[32] M. Frank, Ö. Özdal and P. Poulose, Relaxing LHC constraints on the $W_{R}$ mass, Phys. Rev. D 99 (2019) 035001 [arXiv: 1812.05681] [INSPIRE].

[33] A. Alloul et al., FeynRules 2.0 - A complete toolbox for tree-level phenomenology, Comput. Phys. Commun. 185 (2014) 2250 [arXiv:1310.1921] [inSPIRE].

[34] N.G. Deshpande and E. Ma, Pattern of symmetry breaking with two Higgs doublets, Phys. Rev. D 18 (1978) 2574 [INSPIRE].

[35] E. Ma, Verifiable radiative seesaw mechanism of neutrino mass and dark matter, Phys. Rev. D 73 (2006) 077301 [hep-ph/0601225] [INSPIRE].

[36] R. Barbieri, L.J. Hall and V.S. Rychkov, Improved naturalness with a heavy Higgs: an alternative road to LHC physics, Phys. Rev. D 74 (2006) 015007 [hep-ph/0603188] [INSPIRE].

[37] M. Frank, I. Turan and M. Sher, Neutrino masses in effective rank-5 subgroups of $E_{6} I$ : non-SUSY case, Phys. Rev. D 71 (2005) 113001 [hep-ph/0412090] [INSPIRE].

[38] D. Borah, S. Patra and U. Sarkar, TeV scale left right symmetry with spontaneous D-parity breaking, Phys. Rev. D 83 (2011) 035007 [arXiv: 1006.2245] [INSPIRE]. 
[39] M. Ashry, TeV scale left-right symmetric model with minimal Higgs sector, M.Sc. thesis, Cairo University, Cairo, Egypt (2015).

[40] G. Bélanger et al., MicrOMEGAs5.0: freeze-in, Comput. Phys. Commun. 231 (2018) 173 [arXiv: 1801.03509] [INSPIRE].

[41] A. Belyaev, N.D. Christensen and A. Pukhov, CalcHEP 3.4 for collider physics within and beyond the Standard Model, Comput. Phys. Commun. 184 (2013) 1729 [arXiv:1207.6082] [INSPIRE].

[42] C. Degrande et al., UFO - The Universal FeynRules Output, Comput. Phys. Commun. 183 (2012) 1201 [arXiv: 1108.2040] [INSPIRE].

[43] N.D. Christensen et al., A comprehensive approach to new physics simulations, Eur. Phys. J. C 71 (2011) 1541 [arXiv:0906.2474] [INSPIRE].

[44] J. Alwall et al., The automated computation of tree-level and next-to-leading order differential cross sections and their matching to parton shower simulations, JHEP 07 (2014) 079 [arXiv: 1405.0301] [inSPIRE].

[45] R.D. Ball et al., Parton distributions with LHC data, Nucl. Phys. B 867 (2013) 244 [arXiv:1207.1303] [INSPIRE].

[46] T. Sjöstrand et al., An introduction to PYTHIA 8.2, Comput. Phys. Commun. 191 (2015) 159 [arXiv: 1410.3012] [INSPIRE].

[47] DELPHES 3 collaboration, DELPHES 3, a modular framework for fast simulation of a generic collider experiment, JHEP 02 (2014) 057 [arXiv: 1307.6346] [INSPIRE].

[48] M. Cacciari, G.P. Salam and G. Soyez, The anti- $k_{t}$ jet clustering algorithm, JHEP 04 (2008) 063 [arXiv:0802.1189] [INSPIRE].

[49] M. Cacciari, G.P. Salam and G. Soyez, FastJet user manual, Eur. Phys. J. C 72 (2012) 1896 [arXiv:1111.6097] [INSPIRE].

[50] E. Conte, B. Fuks and G. Serret, MadAnalysis 5, a user-friendly framework for collider phenomenology, Comput. Phys. Commun. 184 (2013) 222 [arXiv:1206.1599] [InSPIRE].

[51] F. Ambrogi et al., MadDM v.3.0: a comprehensive tool for dark matter studies, Phys. Dark Univ. 24 (2019) 100249 [arXiv: 1804.00044] [INSPIRE].

[52] P. Bechtle et al., HiggsBounds: confronting arbitrary Higgs sectors with exclusion bounds from LEP and the Tevatron, Comput. Phys. Commun. 181 (2010) 138 [arXiv:0811.4169] [INSPIRE].

[53] P. Bechtle et al., HiggsSignals: confronting arbitrary Higgs sectors with measurements at the Tevatron and the LHC, Eur. Phys. J. C 74 (2014) 2711 [arXiv:1305.1933] [InSPIRE].

[54] A. Buckley, PySLHA: a Pythonic interface to SUSY Les Houches Accord data, Eur. Phys. J. C 75 (2015) 467 [arXiv: 1305.4194] [INSPIRE].

[55] P.Z. Skands et al., SUSY Les Houches accord: interfacing SUSY spectrum calculators, decay packages and event generators, JHEP 07 (2004) 036 [hep-ph/0311123] [INSPIRE].

[56] Particle Data Group collaboration, Review of particle physics, Phys. Rev. D 98 (2018) 030001 [INSPIRE].

[57] P.S.B. Dev, R.N. Mohapatra and Y. Zhang, Probing the Higgs sector of the minimal left-right symmetric model at future hadron colliders, JHEP 05 (2016) 174 [arXiv: 1602.05947] [INSPIRE]. 
[58] CMS collaboration, Search for pair-produced resonances decaying to quark pairs in proton-proton collisions at $\sqrt{s}=13$ TeV, Phys. Rev. D 98 (2018) 112014 [arXiv: 1808.03124] [INSPIRE].

[59] CMS collaboration, Search for high-mass resonances in dilepton final states in proton-proton collisions at $\sqrt{s}=13 \mathrm{TeV}$, JHEP 06 (2018) 120 [arXiv: 1803.06292] [INSPIRE].

[60] ATLAS collaboration, Search for new high-mass phenomena in the dilepton final state using $36 \mathrm{fb}^{-1}$ of proton-proton collision data at $\sqrt{s}=13$ TeV with the ATLAS detector, JHEP 10 (2017) 182 [arXiv:1707.02424] [INSPIRE].

[61] ATLAS collaboration, Search for new phenomena in dijet events using $37 \mathrm{fb}^{-1}$ of pp collision data collected at $\sqrt{s}=13$ TeV with the ATLAS detector, Phys. Rev. D 96 (2017) 052004 [arXiv: 1703.09127] [INSPIRE].

[62] A. Arhrib et al., The Higgs potential in the type II seesaw model, Phys. Rev. D 84 (2011) 095005 [arXiv: 1105.1925] [INSPIRE].

[63] ATLAS collaboration, Prospects for searches for heavy $Z^{\prime}$ and $W^{\prime}$ bosons in fermionic final states with the ATLAS experiment at the HL-LHC, ATL-PHYS-PUB-2018-044 (2018).

[64] Planck collaboration, Planck 2013 results. XVI. Cosmological parameters, Astron. Astrophys. 571 (2014) A16 [arXiv:1303.5076] [INSPIRE].

[65] MAGIC, FERMI-LAT collaboration, Limits to dark matter annihilation cross-section from a combined analysis of MAGIC and Fermi-LAT observations of dwarf satellite galaxies, JCAP 02 (2016) 039 [arXiv: 1601.06590] [INSPIRE].

[66] XENON collaboration, Dark matter search results from a one ton-year exposure of XENON1T, Phys. Rev. Lett. 121 (2018) 111302 [arXiv:1805.12562] [INSPIRE].

[67] CMS collaboration, Search for heavy stable charged particles with $12.9 \mathrm{fb}^{-1}$ of 2016 data, CMS-PAS-EXO-16-036 (2016).

[68] CMS collaboration, Search for long-lived charged particles in proton-proton collisions at $\sqrt{s}=13$ TeV, Phys. Rev. D 94 (2016) 112004 [arXiv:1609.08382] [INSPIRE].

[69] ATLAS collaboration, Search for long-lived, massive particles in events with a displaced vertex and a muon with large impact parameter in pp collisions at $\sqrt{s}=13$ TeV with the ATLAS detector, arXiv:2003.11956 [INSPIRE].

[70] ATLAS collaboration, Search for heavy charged long-lived particles in the ATLAS detector in $36.1 \mathrm{fb}^{-1}$ of proton-proton collision data at $\sqrt{\mathrm{s}}=13 \mathrm{TeV}$, Phys. Rev. D 99 (2019) 092007 [arXiv: 1902.01636] [INSPIRE].

[71] ATLAS collaboration, Search for long-lived, massive particles in events with displaced vertices and missing transverse momentum in $\sqrt{s}=13$ TeV pp collisions with the ATLAS detector, Phys. Rev. D 97 (2018) 052012 [arXiv:1710.04901] [InSPIRE].

[72] J. Alimena et al., Searching for long-lived particles beyond the standard model at the Large Hadron Collider, arXiv: 1903.04497 [INSPIRE].

[73] CMS collaboration, Searches for long-lived charged particles in pp collisions at $\sqrt{s}=7$ and 8 TeV, JHEP 07 (2013) 122 [arXiv:1305.0491] [INSPIRE].

[74] CMS collaboration, Search for heavy stable charged particles in pp collisions at $\sqrt{s}=7$ TeV, JHEP 03 (2011) 024 [arXiv: 1101.1645] [INSPIRE].

[75] ATLAS collaboration, Search for heavy long-lived charged particles with the ATLAS detector in pp collisions at $\sqrt{s}=7$ TeV, Phys. Lett. B 703 (2011) 428 [arXiv:1106.4495] [InSPIRE]. 
[76] OPAL collaboration, Search for stable and longlived massive charged particles in $e^{+} e^{-}$ collisions at $\sqrt{s}=130$ GeV-183 GeV, Phys. Lett. B 433 (1998) 195 [hep-ex/9803026] [INSPIRE].

[77] CMS collaboration, Search for charged Higgs bosons in the $H^{ \pm} \rightarrow \tau^{ \pm} \nu_{\tau}$ decay channel in proton-proton collisions at $\sqrt{s}=13 \mathrm{TeV}$, JHEP 07 (2019) 142 [arXiv:1903.04560] [INSPIRE].

[78] ATLAS collaboration, Search for charged Higgs bosons produced in association with a top quark and decaying via $H^{ \pm} \rightarrow \tau \nu$ using pp collision data recorded at $\sqrt{s}=13$ TeV by the ATLAS detector, Phys. Lett. B 759 (2016) 555 [arXiv:1603.09203] [INSPIRE].

[79] CMS collaboration, Search for charged Higgs bosons decaying into a top and a bottom quark in the all-jet final state of pp collisions at $\sqrt{s}=13$ TeV, arXiv:2001.07763 [INSPIRE].

[80] ATLAS collaboration, Search for charged Higgs bosons decaying into top and bottom quarks at $\sqrt{s}=13$ TeV with the ATLAS detector, JHEP 11 (2018) 085 [arXiv:1808.03599] [INSPIRE].

[81] ALEPH, DELPHI, L3, OPAL, LEP collaboration, Search for charged Higgs bosons: combined results using LEP data, Eur. Phys. J. C 73 (2013) 2463 [arXiv:1301.6065] [INSPIRE].

[82] B. Dumont et al., Toward a public analysis database for LHC new physics searches using MADANALYSIS 5, Eur. Phys. J. C 75 (2015) 56 [arXiv:1407.3278] [InSPIRE].

[83] E. Conte and B. Fuks, Confronting new physics theories to LHC data with MADANALYSIS 5, Int. J. Mod. Phys. A 33 (2018) 1830027 [arXiv:1808.00480] [INSPIRE].

[84] J.Y. Araz, M. Frank and B. Fuks, Reinterpreting the results of the LHC with MadAnalysis 5: uncertainties and higher-luminosity estimates, arXiv:1910.11418 [INSPIRE].

[85] CMS collaboration, Search for electroweak production of charginos and neutralinos in multilepton final states in proton-proton collisions at $\sqrt{s}=13$ TeV, JHEP 03 (2018) 166 [arXiv: 1709.05406] [INSPIRE].

[86] A. Chatterjee et al., Multileptonic signals of co-annihilating left-right supersymmetric dark matter, Phys. Rev. D 99 (2019) 035017 [arXiv: 1810.03891] [INSPIRE].

[87] B. Fuks and S. Mondal, Search for electroweak production of charginos and neutralinos in multilepton final states in proton-proton collisions at $\sqrt{s}=13$ TeV, CMS-SUS-16-039 (2017).

[88] CMS collaboration, Search for top squarks and dark matter particles in opposite-charge dilepton final states at $\sqrt{s}=13$ TeV, Phys. Rev. D 97 (2018) 032009 [arXiv:1711.00752] [INSPIRE].

[89] B. Fuks et al., Proceedings of the first MadAnalysis 5 workshop on LHC recasting in Korea, arXiv: 1806.02537.

[90] S. Bein et al., Search for top squarks and dark matter particles in opposite-charge dilepton final states at $\sqrt{s}=13$ TeV, CMS-SUS-17-001 (2017).

[91] G. Cowan, K. Cranmer, E. Gross and O. Vitells, Asymptotic formulae for likelihood-based tests of new physics, Eur. Phys. J. C 71 (2011) 1554 [Erratum ibid. C 73 (2013) 2501] [arXiv: 1007.1727] [INSPIRE]. 